\title{
Integrating single-cell sequencing data with GWAS summary statistics reveals CD16+monocytes and memory CD8+T cells involved in severe COVID-19
}

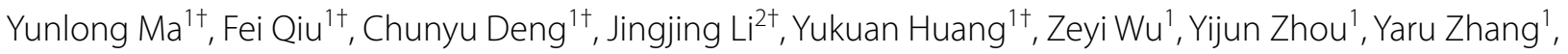
Yichun Xiong ${ }^{3}$, Yinghao Yao ${ }^{3}$, Yigang Zhong ${ }^{4}$, Jia Qu ${ }^{1}$ and Jianzhong Su ${ }^{1,3^{*}}$ (⿺)

\begin{abstract}
Background: Understanding the host genetic architecture and viral immunity contributes to the development of effective vaccines and therapeutics for controlling the COVID-19 pandemic. Alterations of immune responses in peripheral blood mononuclear cells play a crucial role in the detrimental progression of COVID-19. However, the effects of host genetic factors on immune responses for severe COVID-19 remain largely unknown.

Methods: We constructed a computational framework to characterize the host genetics that influence immune cell subpopulations for severe COVID-19 by integrating GWAS summary statistics ( $N=969,689$ samples) with four independent scRNA-seq datasets containing healthy controls and patients with mild, moderate, and severe symptom ( $N=606,534$ cells). We collected 10 predefined gene sets including inflammatory and cytokine genes to calculate cell state score for evaluating the immunological features of individual immune cells.

Results: We found that 34 risk genes were significantly associated with severe COVID-19, and the number of highly expressed genes increased with the severity of COVID-19. Three cell subtypes that are CD16+monocytes, megakaryocytes, and memory CD8+T cells were significantly enriched by COVID-19-related genetic association signals. Notably, three causal risk genes of $C C R 1, C X C R 6$, and $A B O$ were highly expressed in these three cell types, respectively. $C C R 1^{+} \mathrm{CD} 16+$ monocytes and $A B \mathrm{O}^{+}$megakaryocytes with significantly up-regulated genes, including $S 100 \mathrm{~A} 12$, S100A8, S100A9, and IFITM1, confer higher risk to the dysregulated immune response among severe patients. CXCR6 ${ }^{+}$ memory CD8+ T cells exhibit a notable polyfunctionality including elevation of proliferation, migration, and chemotaxis. Moreover, we observed an increase in cell-cell interactions of both $C C R 1^{+} \mathrm{CD} 16+$ monocytes and $C X C R 6^{+}$memory CD8+T cells in severe patients compared to normal controls among both PBMCs and lung tissues. The enhanced interactions of $C X C R 6^{+}$memory CD8+T cells with epithelial cells facilitate the recruitment of this specific population of T cells to airways, promoting CD8+T cell-mediated immunity against COVID-19 infection.
\end{abstract}

\footnotetext{
*Correspondence: sujz@wmu.edu.cn

${ }^{\dagger}$ Yunlong Ma, Fei Qiu, Chunyu Deng, Jingjing Li and Yukuan Huang contributed equally to this work.

${ }^{3}$ Wenzhou Institute, University of Chinese Academy of Sciences,

Wenzhou 325011, China

Full list of author information is available at the end of the article
}

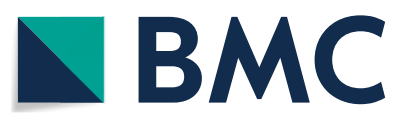

(c) The Author(s) 2022. Open Access This article is licensed under a Creative Commons Attribution 4.0 International License, which permits use, sharing, adaptation, distribution and reproduction in any medium or format, as long as you give appropriate credit to the original author(s) and the source, provide a link to the Creative Commons licence, and indicate if changes were made. The images or other third party material in this article are included in the article's Creative Commons licence, unless indicated otherwise in a credit line to the material. If material is not included in the article's Creative Commons licence and your intended use is not permitted by statutory regulation or exceeds the permitted use, you will need to obtain permission directly from the copyright holder. To view a copy of this licence, visit http://creativecommons.org/licenses/by/4.0/. The Creative Commons Public Domain Dedication waiver (http://creativeco mmons.org/publicdomain/zero/1.0/) applies to the data made available in this article, unless otherwise stated in a credit line to the data. 
Conclusions: We uncover a major genetics-modulated immunological shift between mild and severe infection, including an elevated expression of genetics-risk genes, increase in inflammatory cytokines, and of functional immune cell subsets aggravating disease severity, which provides novel insights into parsing the host genetic determinants that influence peripheral immune cells in severe COVID-19.

Keywords: Single-cell sequencing, GWAS, Immune cells, Inflammatory storm, COVID-19

\section{Background}

The coronavirus disease 2019 (COVID-19) outbreak, caused by severe acute respiratory syndrome coronavirus 2 (SARS-CoV-2), has widely and severely jeopardized the health and economy systems of most countries worldwide. As of January 26, 2022, there were more than 359.1 million confirmed patients with more than 5.63 million deaths in the whole world [1]. COVID-19 has distinct clinical manifestations ranging from asymptomatic to severe respiratory failure [2]. Mortalities of COVID-19 are largely derived from severe patients with interstitial pneumonia in both lungs and acute respiratory distress syndrome [3]. Many earlier studies [4-6] have shown that the number of severe COVID-19 patients who are elders and have comorbidities, such as diabetes and hypertension, has increased. In this connection, understanding the immunologic mechanism of severe COVID-19 and identifying novel vaccine targets to control the pandemic are of considerable interest.

Accumulating evidence has suggested that alterations of immune responses in peripheral blood mononuclear cells (PBMCs) and bronchoalveolar lavage fluid (BALF) play a crucial role in the detrimental progression of COVID-19 [7, 8]. There has been evidence that cytokine storm, usually found in severe COVID-19 patients, causes the adverse progression of COVID-19 [7]. Increased circulating levels of proinflammatory cytokine, including IL-10, IL-6, and TNF- $\alpha$, have been reported to be associated with severe COVID-19 [7, 9]. Single-cell RNA sequencing (scRNA-seq) has been extensively utilized to reveal the immune responses of COVID-19 patients in both lung and peripheral blood [10-18]. Megakaryocytes and monocytes [11, 12], T cell exhaustion [14], lymphopenia [19], and increased levels of cytokines [20] may cause aberrant peripheral immune activities in severe COVID-19 patients. Based on large-scale samples, previous studies identified that dysregulation of the mTOR signaling pathway in dendritic cells [21] and aberrant myeloid cell subpopulations $[16,17]$ implicated in severe COVID-19. Su et al. [10] revealed an increase in inflammation and a sharp drop in blood nutrients between mild and moderate-to-severe COVID-19, and new subsets of immune cells emerged in moderate COVID-19 patients.

Genome-wide association study (GWAS) has emerged as a powerful approach to identify risk genes and genetic variants for complex diseases. By gathering population-based GWAS data worldwide, the COVID-19 Host Genetic Consortium has launched the "COVID-19 Host Genetics Initiative" project to facilitate COVID-19 host genetic research and identify genetic determinants of COVID-19 [22]. Subsequently, a growing number of GWASs have identified numerous significant genetic variants associated with COVID-19 susceptibility and severity [23-28]. Ellinghaus et al. [27] performed a meta-analysis of two independent GWAS datasets with 1610 severe COVID-19 patients and 2205 matched controls at seven hospitals in the Italian and Spanish epicenters and identified two susceptibility loci of 3p21.31 and 9q34.2 to be significantly associated with severe COVID-19 at the genome-wide level. Based on a large-scale meta-analysis $(N=680,128)$, our group found that the IFNAR2-IL1ORB gene cluster was significantly associated with COVID-19 susceptibility, and suggested that IFNAR2 and IL1ORB might have regulatory roles in the pulmonary immune response based on scRNA-seq data [25]. Consistently, Pairo-Gastineira et al. [24] conducted a GWAS study based on 2244 critically ill COVID-19 patients and highlighted that several genes including IFNAR2, DPP9, and OAS1 were significantly associated with severe COVID-19 at a genome-wide significance.

Two primary hypotheses were proposed for the involvement of immune genes in severe COVID-19 susceptibility: whether the severe COVID-19-related risk genes associated with defective innate immune responses would induce persistent viral replication and resultant high viral loads, and whether an exaggerated genetically mediated cytokine production contributes to the hyper-inflammation and poor outcome among severe COVID-19. However, the effects of these genetic determinants on the peripheral immune cells for severe COVID-19 remain largely unknown. In view of a purely genetic study or single-cell sequencing study cannot address this critical question, we here leveraged comprehensive computational methods to combine a largescale GWAS summary dataset with scRNA-seq data for identifying host genetics that influence immune cell subpopulations involved in the etiology of severe COVID-19. 


\section{Methods}

\section{Single-cell RNA-seq data on severe COVID-19}

In this study, we downloaded four independent scRNAseq datasets on COVID-19 in PBMC and BALF from the ArrayExpress database (dataset \#1, the accession number is E-MTAB-9357 from Su et al. study [10]) and the Gene Expression Omnibus (GEO) database (dataset \#2, the accession number is GSE149689 from Lee et al. study [18]; dataset \#3, the accession number is GSE150861 from Guo et al. study [11]; and dataset \#4, the accession number is GSE158055 [12]). The first dataset contained 270 peripheral blood samples including 254 samples with different COVID-19 severity (i.e., mild $N=109$, moderate $N=102$, and severe $N=50$ ) and 16 healthy controls for scRNA-seq analysis. There were eight patients in dataset \#2 with COVID-19 of varying clinical severity, including asymptomatic, mild, and severe, and four healthy controls with PBMCs. Dataset \#3 included five peripheral blood samples collected from two severe COVID-19 patients at three different time points during tocilizumab treatment, containing two different stages: severe stage and remission stage. Within dataset \#4, 12 BALF samples were collected from lung tissues, including three moderate and nine severe patients. For all the datasets, the sample collection process was reviewed and approved by Institutional Review Boards at the institutions where samples were originally collected. As performed in the original studies [10-12, 18], the COVID-19 severity was evaluated by using the World Health Organization (WHO) ordinal scale (WOS), the National Early Warning Score (NEWS), or the Diagnosis and Treatment of COVID-19 (Trial Version 6). Single-cell transcriptomes for these four datasets were gathered using the $10 \times$ Genomics scRNA-seq platform [29]. A total of 606,534 cells with 563,856 PBMC cells and 42,678 BALF cells were yielded from 300 samples based on the four independent scRNA-seq datasets. To allow comparison across samples and datasets, we used a common dictionary of gene symbols to annotate genes and these unrecognized symbols were removed.

\section{Single-cell RNA sequencing data processing}

We performed normalization, clustering, and dimensionality reduction; differential expression gene (DEG) analysis; and visualization on these four independent scRNA-seq datasets with the Seurat R package [30]. The SCTransform function was used to scale and transform data, and a linear regression model was applied to omit redundant variations caused by cellular complexity (i.e., cells expressed less than 200 genes or more than 2500 genes were removed) or cellular quality (i.e., cells that had UMIs more than 10,000 and expressed reads of mitochondrial genes greater than $10 \%$ were removed). The CellCycleSoring function was applied to remove the effects of confounding factors. Principal component analysis (PCA) was carried out to extract principal components (PCs) that could explain most of the datasets via using high variable genes. Top 20 PCs were utilized to conduct uniform manifold approximation and projection (UMAP) to embed the dataset into two dimensions. Subsequently, we constructed a shared nearest-neighbor graph (SNN) using the FindNeighbors function based on the top 20 PCs and applied a graph-based modularity-optimization algorithm from the Louvain method [31] on this SNN for clustering the dataset with the cluster resolution set to 0.5 . We used the RunHarmony function with the PCA reduction method from harmony $\mathrm{R}$ package [32] to integrate samples to correct batch effects. The FindConservedMarkers function in Seurat was implemented to find differential expressed genes for determining cellular identity. Well-defined markers were used to annotate clusters, and uncharacterized clusters in the first round of clustering were extracted to run the second round of clustering.

\section{GWAS summary data on hospitalized COVID-19}

The meta-GWAS summary data on severe COVID-19 round 4 (B2_ALL, Susceptibility [Hospitalized COVID19 vs. Population]) were downloaded from the official website of the COVID-19 Host Genetic Consortium [22] (https://www.covid19hg.org/; analyzed file named: “COVID19_HGI_B2_ALL_leave_23andme_20201020. txt.gz"; released date of October 4, 2020). There were 7885 hospitalized COVID-19 patients and 961,804 control participants from 21 independent contributing studies. There was an overwhelming majority of participants in these contributing studies with European ancestry (93\%). The meta-GWAS summary statistics contained $P$ values, Wald statistic, inverse-variance meta-analyzed log odds ratio (OR), and related standard errors. The 1,000 Genomes Project European Phase 3 [33] was used as a panel for pruning. Results from 23\&Me cohort GWAS summary statistics were excluded from our current analysis. Genetic variants without RefSNP number in the Human Genome reference builds 37 were filtered out, giving a total of 9,368,170 genetic variants satisfying the major allele frequency (MAF) over 0.0001 and the imputation score of greater than 0.6. We used the qqman $\mathrm{R}$ package to figure both Manhattan plot and quantile-quantile (QQ) plot, and the Web-based software of LocusZoom (http://locuszoom.sph.umich.edu/) [34] to visualize the regional association plots for significant risk loci (see Additional file 1: Supplementary methods). 


\section{Hierarchical clustering analysis}

To examine the similarity of the transcriptome profiles between cell types across different COVID-19 severities, we merged the counts of UMI for each cell type according to normal, mild, moderate, and severe COVID-19. In order to normalize gene expression, we divided the counts of UMI for each gene by the counts of total UMI for all genes in each cell type and then multiplied by 100,000 , as refer to the method in a previous study [18]. Based on a median expression value of greater than 0.5 , we calculated the relative changes in gene expression divided by the median value for each gene. The Pearson correlation coefficient (PPC) of the relative change in gene expression was used for the current hierarchical clustering analysis.

\section{Gene-based association analysis}

To perform a gene-based genetic association analysis of the meta-GWAS summary statistics on severe COVID19 , we leveraged the updated SNP-wise mean model of MAGMA [35]. In this model, MAGMA computes a test statistic:

$$
T=\sum_{i}^{N} Z_{i}^{2}=Z^{T} \boldsymbol{Z}
$$

where $N$ is the number of SNPs mapped in a gene and $Z_{i}=\varphi\left(p_{i}\right)$. Of note, $\varphi$ is the cumulative normal distribution function and $p_{i}$ is the marginal $P$ value for a given SNP $i$. SNPs belonging to a specific gene were based on whether located in the gene body or within the $\pm 20 \mathrm{~kb}$ upstream or downstream region of the gene. Furthermore, the model assumes $\boldsymbol{Z} \sim \operatorname{MVN}(\boldsymbol{O}, \boldsymbol{S})$, where $\boldsymbol{S}$ is the LD matrix of the SNP genotypes. The LD matrix can be diagonalized and hence written as $\boldsymbol{S}=\boldsymbol{Q} \boldsymbol{A} \boldsymbol{Q}^{T}$, where $\boldsymbol{Q}$ is an orthogonal matrix and $\boldsymbol{A}=\operatorname{diag}\left(\lambda_{1}, \lambda_{2}, \ldots, \lambda_{N}\right)$ with $\lambda_{j}$ being the $j$ th eigenvalue of $S$. The 1,000 Genomes Project Phase 3 European Panel [33] was used for calculating the LD information among SNPs extracted from GWAS summary data on COVID-19.D $\operatorname{MVN}\left(\boldsymbol{O}, \boldsymbol{I}_{K}\right)$ is a random variable, where $D=A^{-0.5} Q^{T} Z$. Then, the sum of squared SNP $Z$-statistics as the following formula:

$$
T=\boldsymbol{Z}^{T} \boldsymbol{Z}=\left(\boldsymbol{Q} A^{0.5} \boldsymbol{D}\right)^{T} \boldsymbol{Q} \boldsymbol{A}^{\mathbf{0 . 5}} \boldsymbol{D}=\boldsymbol{D}^{T} \boldsymbol{A} \boldsymbol{D}=\sum_{i}^{N} \lambda_{i} D_{i}^{2}
$$

with $D_{i} \sim \mathrm{N}(0,1)$ and $D_{i}^{2} \sim \chi_{1}^{2}$. Namely, $T$ follows a mixture distribution of independent $\chi_{1}^{2}$ random variables. A total of 19,138 genes were included in the current analysis. We used the Benjamini-Hochberg false discovery rate (FDR) method, in which a gene with a FDR $\leq 0.05\left(P \leq 6.8 \times 10^{-5}\right)$ was interpreted as significant, to adjust for multiple testing.

\section{Pathway enrichment analysis}

We applied the built-in functions of MAGMA [35], using the results from GWAS summary statistics as its input, to examine genome-wide enriched biological pathways for severe COVID-19. We calculated competitive $P$ values by examining the results that the combined effect of genes within a pathway is significantly greater than the combined effect of all other genes, and 10,000 permutations were used to adjust competitive $P$ values. Additionally, we leveraged the over-representation algorithm of the WebGestalt (http://www.webgestalt. org) [36] along with the significant genes as an input list to conduct a pathway enrichment analysis using the KEGG pathway resource [37]. The number of genes in each pathway was set to between 5 and 2000, and the Benjamini-Hochberg FDR was used for multiple correction. To cluster these identified KEGG pathways, we performed a multidimensional scaling (MDS) analysis based on the Jaccard distance method [38,39] and constructed a pathway-pathway interaction network for these significantly enriched pathways setting the Jaccard distance > 0.1 . For the analyzed codes, please refer to the GitHub repository (https://github.com/mayunlong89/COVID 19_scRNA [40]).

\section{Combining GWAS-based genetic signals with eQTL data}

To uncover genetically regulatory expression of genes associated with severe COVID-19, we conducted an integrative genomics analysis by using the S-PrediXcan [41] by combining meta-GWAS summary statistics with expression quantitative trait loci (eQTL) data for 49 tissues from the GTEx Project (version 8) [42]. S-PrediXcan mainly uses two linear regression models to analyze the association between predicted gene expression and severe COVID-19:

$$
\begin{aligned}
& \boldsymbol{Y}=\boldsymbol{\alpha}_{1}+\boldsymbol{X}_{l} \beta_{l}+\boldsymbol{\varepsilon}_{1} \\
& \boldsymbol{Y}=\boldsymbol{\alpha}_{2}+\boldsymbol{G}_{g} \gamma_{g}+\boldsymbol{\varepsilon}_{2}
\end{aligned}
$$

where $\boldsymbol{\alpha}_{1}$ and $\boldsymbol{\alpha}_{2}$ are intercepts, $\boldsymbol{\varepsilon}_{1}$ and $\boldsymbol{\varepsilon}_{2}$ are independent error terms, $\boldsymbol{Y}$ is the $n$-dimensional vector for $n$ individuals, $X_{l}$ is the allelic dosage for SNP $l$ in $n$ individuals, $\beta_{l}$ is the effect size of SNP $l, G_{g}=\sum_{i \in \text { gene(g) }} \omega_{i g} X_{i}$ is the predicted expression calculated by $\omega_{l g}$ and $\boldsymbol{X}_{l}$, in which $\omega_{l g}$ is derived from the GTEx Project, and $\gamma_{g}$ is the effect size of $\mathrm{G}_{g}$. The $Z$-score (Wald-statistic) of the association between predicted gene expression and severe COVID19 can be transformed as:

$$
Z_{g}=\frac{\hat{\gamma}_{g}}{\operatorname{se}\left(\hat{\gamma}_{g}\right)} \approx \sum_{i \in \text { gene }(g)} \omega_{i g} \frac{\hat{\sigma}_{i}}{\hat{\sigma}_{g}} \frac{\hat{\beta}_{i}}{\operatorname{se}\left(\hat{\beta}_{i}\right)}
$$


where $\hat{\sigma}_{g}$ is the standard deviation of $\mathrm{G}_{g}$ and can be calculated from the 1,000 Genomes Project European Phase 3 Panel, $\hat{\beta}_{l}$ is the effect size from GWAS on COVID-19, and $\hat{\sigma}_{l}$ is the standard deviation of $\hat{\beta}_{l}$. S-PrediXcan was run for each of 49 tissues with 659,158 gene-tissue pairs.

Furthermore, to increase the power to discover significant genes whose expression has similar regulations across multi-tissues, we utilized the S-MultiXcan [43] to meta-analyze these results from the above S-PrediXcan analysis. S-MultiXcan fits a linear regression model of severe COVID-19 on predicted expression from multiple tissue models jointly:

$$
\mathrm{Y}=\sum_{j=1}^{p} \mathrm{~T}_{j} g_{j}+\mathrm{e}=\mathrm{Tg}+\mathrm{e}
$$

where $\tilde{\mathrm{T}}_{j}=\sum_{i \in \text { gene }(j)} \omega_{i} \mathrm{X}_{i}$ is the predicted expression of tissue $j$ and $\mathrm{T}_{j}$ is the standardization of $\tilde{\mathrm{T}}_{j}$ to mean $=0$ and standard deviation $=1 . g_{j}$ is the effect size for the predicted gene expression in tissue $j$, e is an error term with variance $\sigma_{e}^{2}$, and $p$ is the number of included tissues. There were 22,326 genes across 49 GTEx tissues with integrated convergent evidence in S-MultiXcan, and a gene with a value of FDR $\leq 0.05\left(P \leq 3.8 \times 10^{-5}\right)$ is considered to be significant.

\section{In silico permutation analysis}

To explore the concordance of results from both MAGMA analysis (gene set $\# 1: N=944, P \leq 0.05$ ) and S-MultiXcan analysis (gene set $\# 2: N=1274, P \leq 0.05$ ), we performed an in silico permutation analysis which consisted 100,000 times $\left(N_{\text {Total }}\right)$ random selections [44, 45]. We first calculated the number of overlapped genes between gene sets \#1 and \#2 $\left(N_{\text {Observation }}=302\right)$, then employed the total number of genes in S-MultiXcan analysis as background genes $\left(N_{\text {Background }}=22,326\right)$. By randomly selecting the same number of genes as gene set $\# 2(N=1274)$ from the background genes, and after repeating it 100,000 times, we calculated the number of overlapped genes between gene set \#1 and the sample we selected each time $\left(N_{\text {Random }}\right)$. Finally, we calculated the empirically permuted $P$ value using the following formula: $P=\frac{N_{\text {Random }} \geq N_{\text {Observation }}}{N_{\text {Total }}}$, and empirical $P$ value $\leq 0.05$ is considered to be significant.

\section{Drug-gene interaction analysis}

We conducted a drug-gene interaction analysis for identified genetics-risk genes by using protein-chemical interactions in the context of STRING-based PPI networks [46] and STITCH-based drug annotation information (v5.0, http://stitch.embl.de/) [47]. Only experimentally validated gene-drug interactions with ranked confidence score were selected for constructing a drug-gene interaction network. To examine the potential therapeutic effects of highly expressed genes in each immune cell, we conducted an enrichment analysis of 43 druggable categories based on the DGIdb database (https://www.dgidb.org/druggable_gene_ categories) [48]. Additionally, we collected 1263 human druggable proteins, which are therapeutic targets of clinical stage or approved drugs, from a previous study [26]. Among them, 704 proteins are targets for potential COVID-19-relevant drugs based on registers of clinical trials for COVID-19, approved immunomodulatory/anticoagulant drugs, or have biological functions associated with SARS-CoV-2 infection.

\section{Integrated analysis of GWAS summary statistics and scRNA-seq data}

To identify genetically regulatory-related peripheral immune cells for severe COVID-19, we implemented the RolyPoly algorithm [49] to incorporate GWAS summary statistics with scRNA-seq data. Let $g(i)$ stand for the gene associated with SNP $i, S_{j}=\{i: g(i)=j\}$ be the SNP set with multiple SNPs associated with the gene $j$, and $\beta_{S_{j}}$ be a GWAS-based effect-size vector of $S_{j}$ with a priori assumption that $\beta_{S_{j}} \sim \operatorname{MVN}\left(\mathbf{0}, \sigma_{\mathrm{j}}^{2} \boldsymbol{I}_{\left|S_{j}\right|}\right)$. Following the prior, RolyPoly gives a polygenic linear model for $\beta_{S_{j}}$ :

$$
\sigma_{j}^{2}=\gamma_{0}+\sum_{i=1}^{N} \gamma_{i} \alpha_{j i}
$$

where $\gamma_{0}$ is an intercept term, $\alpha_{j i}(i=1,2, \ldots, N)$ are annotations such as cell-type-specific gene expression, and $\gamma_{i}$ are annotation coefficients for $\alpha_{j i}$. To fit the observed and expected sum squared SNP effect sizes related to each gene by using the method-of-moments estimators, RolyPoly estimates $\gamma_{i}$ by the following equation:

$$
E\left(\sum_{i \in S_{j}} \hat{\beta}_{i}^{2}\right)=\sigma_{j}^{2} \operatorname{Tr}\left(\mathrm{R}_{S_{j}}^{2}\right)+\left|S_{j}\right| \sigma_{e}^{2} n^{-1}
$$

where $\mathrm{R}_{S_{j}}$ is the LD matrix of $S_{j}$ and $\mathrm{Tr}$ represents the trace of a matrix. Finally, RolyPoly applies the block bootstrap method with 1000 iterations to estimate standard errors $\hat{\sigma}_{\gamma_{i}}$ for calculating a $t$-statistic and corresponding $P$ values. The PLINK (v1.90) [50] was used to calculate the LD between SNPs within the $1-\mathrm{Mb}$ window based on the 1,000 Genome Project European Phase 3 panel [33]. We restricted the analysis to SNPs in the autosomes, and any SNPs with MAF $\leq 5 \%$ were 
excluded. The major histocompatibility complex region (Chr6: 25-35 Mbp) was also excluded due to the extensive $\mathrm{LD}$ in this region.

\section{Defining cell state scores}

We leveraged cell state score (CTS) to evaluate the immunological degree of individual immune cells expressed a certain predefined expression gene set $[12,14,51]$. The CTSs were initially depended on the average expression of the genes from the predefined gene set in the respective cell. For a given cell $m$ and a gene set $k\left(G_{k}\right)$, the cell state score $\mathrm{CTS}_{k}(m)$ was defined as the average relative expression (RE) of the genes in $\mathrm{GS}_{k}$. Nevertheless, such initial scores may be confounded by cell complexity, as cells with higher complexity have more genes identified and consequently would be expected to obtain higher cell state scores for any expression gene set. To control for this confounding effect by adding a control gene set $\left(\mathrm{CG}_{k}\right)$, we calculate a similar cell state score with the control gene set and subtract it from the initial cell scores: $\operatorname{CTS}_{k}(m)=$ average $\left[\operatorname{RE}\left(\mathrm{GS}_{k}, m\right)\right]-$ average$\left[\operatorname{RE}\left(\mathrm{CG}_{k}, m\right)\right]$. The control gene set was randomly chosen on the basis of aggregate expression level bins, which obtain a comparable distribution of expression levels and over size to that of the pre-curated gene set The AddModuleScore function in Seurat [30] was applied to calculate the CTS with default parameters.

We used the inflammatory and cytokine genes $(N=$ 324 genes), cytokine-cytokine receptor interactions $(N=294$ genes $)$, chemokine signaling pathway $(N=$ 189 genes), T cell activation (GO: 0042110), response to interferon alpha (GO: 0035455), response to interferon beta (GO: 0035456), leukocyte migration (GO: 0050900), 5 well-defined proliferating markers (MK167, TYMS, NKG7, IL7R, and CCR7), 6 well-defined exhaustion markers (LAG3, TIGIT, PDCD1, CTLA4, HAVCR2, and $T O X$ ), and 12 cytotoxicity-associated genes (PRF1, IFNG, GNLY, NKG7, GZMB, GZMA, GZMH, KLRK1, $K L R B 1, K L R D 1, C T S W$, and CST7) to define inflammatory cytokine, chemokine, $\mathrm{T}$ cell activation, IFN$\alpha / \beta$ response, migration, proliferation, exhaustion, and cytotoxicity score, respectively. These two gene sets of inflammatory and cytokine genes $(N=324$ genes $)$ and cytokine-cytokine receptor interactions $(N=294$ genes $)$ were collected to evaluate the level of hyperinflammatory response, which is used to reflect the degree of "cytokine storm" [52].

\section{Cell-to-cell interaction analysis}

To identify potential cellular interactions of $C C R 1^{+}$ CD16+monocytes and $\mathrm{CXCR6}^{+}$memory $\mathrm{CD} 8+\mathrm{T}$ cells with other immune cells, we utilized the CellChat $\mathrm{R}$ package [53] for inferring the predicted cell-to-cell communications based on two normalized scRNA-seq datasets (dataset \#1 of PBMC and dataset \#4 of BALF). CellChat algorithm could examine the significance of ligand-receptor interactions between two cell types depending on the expression of important factors, including stimulatory and inhibitory membrane-bound co-receptors, soluble agonists, and antagonists. The communication probability of a signaling pathway was derived from the sum of probabilities of their ligandreceptor interactions. We only concentrated on the ligand-receptor interactions that were significantly associated with severe COVID-19 compared with normal control.

\section{Compositional analysis for the proportions of immune cells using the scCODA method}

To validate our findings concerning the different percentage of each cell type in PBMCs, we leveraged a Bayesian model of scCODA [54] for re-conducting the compositional single-cell data analysis. The scCODA framework modeled cell type counts with a hierarchical DirichletMultinomial distribution that accounts for the uncertainty in the proportions of immune cell types in PBMCs and the negative correlative bias via collectively modelling of all measured immune cell type proportions rather than individual ones. The Bayesian inference model applied a Logit-normal spike-and-slab prior [55] with a log-link function to estimate these continuous or binary covariates' effects on immune cell type proportions in PBMCs in a parsimonious fashion. In light of compositional analysis need a reference for identifying compositional changes [56], we used the default parameter in the scCODA: reference_cell_type = "automatic".

\section{Statistical analysis}

The Wilcoxon sum-rank test was used to assess DEGs in mild, moderate, and severe COVID-19 groups compared with normal control [40]. Both the Wilcoxon sumrank test and scCODA were used for composition data analysis to examine the different proportions of peripheral immune cells across different COVID-19 severity. The Mann-Kendall trend analysis was applied to evaluate the significance of cell state cells with elevated severities of COVID-19. Pathway- and disease-based enrichment analyses used the hypergeometric test to identify remarkable biological pathways and disease terms [36]. The Pearson correlation analysis was used to calculate the correlation coefficient of highly expressed genes in $\mathrm{CCR}^{+} \mathrm{CD} 16+$ monocytes between moderate and severe patients [40]. The paired Student's $t$ test was used to calculate the significance of ligand/receptor interactions of $C C R 1^{+} \mathrm{CD} 16+$ monocytes and $C X C R 6^{+}$ 
memory CD8+ T cells with other immune cells between normal control and severe COVID-19.

\section{Results}

The computational framework of the current investigation As shown in Fig. 1, we devised a computational framework to parse the host genetics-modulated immune cell subpopulations implicated in severe COVID-19. It included three main parts: (1) integrative analysis that combined GWAS summary statistics with scRNA-seq data to genetically map single-cell landscape for severe COVID-19 (Fig. 1A and Additional file 2: Table S1); (2) identifying genetics-risk genes, pathways, and immune cell subpopulations that contributed to cytokine storms among severe patients (Fig. 1B); and (3) uncovering the cellular interactions of genetics-modulated immune cell subsets, as well as their functions with cells in lung tissues (Fig. 1C).

\section{Identification of immune cell types associated with severe COVID-19}

To parse the host genetics-influenced immune responses at a single cellular level in PBMCs for severe COVID19 , we subjected three independent scRNA-seq datasets with 563,856 cells to UMAP based on highly variable genes using the Seurat (see the "Methods" section) [30]. There was identification of 13 distinct clusters unbiased by patients with different severities (Additional file 2: Table S2 and Additional file 3: Fig. S1). We leveraged well-known marker genes to assign these clusters to 13 distinct cell types, including mature B cells, megakaryocytes, naïve B cells, CD34+progenitors, dendritic cells, natural killer (NK) cells, CD14+monocytes,

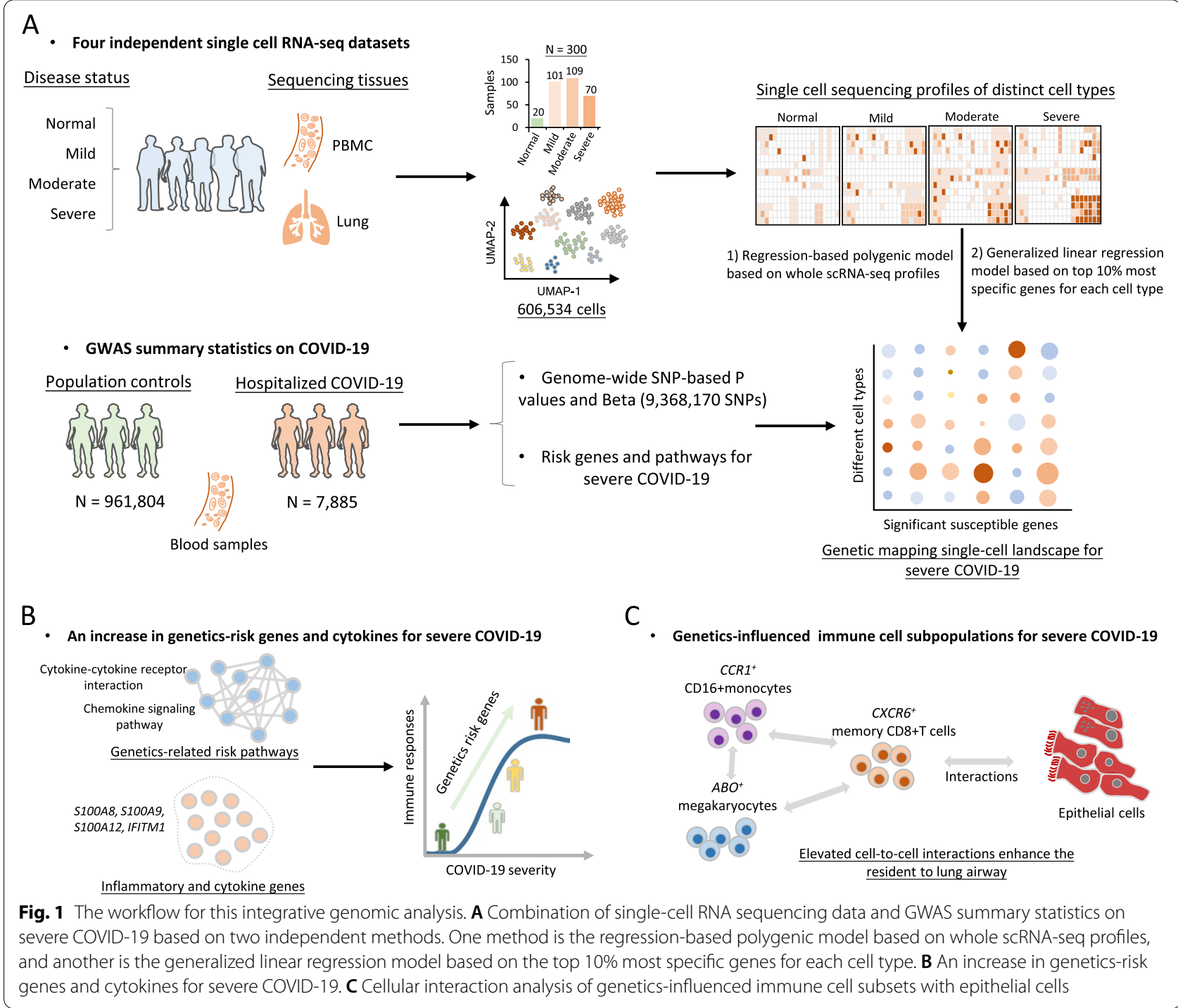


CD16+monocytes, memory CD4+T cells, naïve CD4+T cells, naïve $\mathrm{CD} 8+\mathrm{T}$ cells, memory $\mathrm{CD} 8+\mathrm{T}$ cells, and effector CD8+T cells (Additional file 3: Fig. S2-S3).

While performing the hierarchical clustering analysis on the scRNA-seq profiles, we discovered that cell types were the primary determinants of their clustering, followed by disease severities, indicating both COVID19 pathology and immune cell types might have crucial roles in altered patterns of immune transcriptome instead of technical artifacts (Additional file 3: Fig. S4). As a vital feature for reflecting the alterations of immune responses, we examined the relative proportions of peripheral immune cells across different COVID-19 groups in comparison with the normal group. The proportions of CD14+monocytes, megakaryocytes, and CD34+progenitors were significantly elevated in moderate and severe patients, whereas the proportions of effector CD8+ $\mathrm{T}$ cells, memory CD8+T cells, memory CD4+T cells, naïve CD4+T cells, and NK cells were significantly decreased with the increased severities (Additional file 3: Fig. S5). To provide additional validated evidence, we used an independent method of scCODA to re-perform the compositional data analysis and found the results from scCODA [54] are highly consistent with the above findings from the Wilcoxon sum-rank test (Additional file 3: Fig. S6).

\section{Identification of genetic risk loci associated with severe COVID-19}

Through performing a meta-analysis of 21 independent GWAS studies from the COVID-19 Host Genetic Consortium, eight genomic loci were identified to be associated with hospitalization in COVID-19 patients at a genome-wide significant level, including $1 \mathrm{p} 22.2$ (rs2166172, $\left.P=2.74 \times 10^{-8}\right)$, 3p21.31 (rs35081325, $P=$ $3.32 \times 10^{-58}$, and rs33998492, $\left.P=3.59 \times 10^{-14}\right), 6 \mathrm{p} 21.33$ (rs143334143, $\left.P=1.28 \times 10^{-10}\right)$, 7p11.2 (rs622568, $P=$ $\left.2.57 \times 10^{-8}\right), 9 \mathrm{q} 34.2\left(\mathrm{rs} 505922, P=2.24 \times 10^{-9}\right), 12 \mathrm{q} 24.13$ (rs2269899, $\left.P=3.24 \times 10^{-8}\right), 19 p 13.3(\mathrm{rs} 2109069, P=$ $\left.6.4 \times 10^{-13}\right)$, and $21 \mathrm{q} 22.11\left(\mathrm{rs} 13050728, P=1.91 \times 10^{-11}\right)$ (Fig. 2A, Additional file 2: Table S3 and Additional file 3: Fig. S7). Among these eight loci, three loci, 1p22.2, $6 \mathrm{p} 21.33$, and 7p11.2, were newly identified. Consistently, these eight loci were replicated by using a GWAS with critically ill cases of COVID-19 who needed respiratory support in hospital or who died due to the disease (Additional file 2: Table S4). It should be noted that there were two independent genetic association signals (index SNPs: rs35081325 and rs33998492) in the 3p21.31 locus for severe COVID-19 (Fig. 2B and Additional file 3: Fig. S8). Using the Variant2Gene (V2G) algorithm [57], we prioritized CXCR6 as a candidate causal gene for rs35081325 and causal gene CCR1 for rs33998492 (see Additional file 1: Supplementary methods).

Furthermore, the index SNP of $\operatorname{rs505922~}(P=$ $2.24 \times 10^{-9}$ ) in the $9 \mathrm{q} 34.2$ locus is highly $\mathrm{LD}$ with the reported SNP of $\operatorname{rs657152}\left(R^{2}=0.874\right)$ [27] and rs8176719 $\left(R^{2}=0.876\right)$ [25]. Based on the top-ranked V2G score for rs505922, we prioritized $A B O$ as a potential causal gene contributing susceptibility to severe COVID-19. By performing a MAGMA gene-level association analysis, we observed that 25 genes including CXCR6, CCR1, IFNAR2, IL1ORB, and OAS1 were significantly associated with severe COVID-19 (FDR $<0.05$, Additional file 2: Table S5 and Additional file 3: Fig. S9). GWAS-based pathway enrichment analysis revealed that 19 biological pathways, including cytokine-cytokine receptor interaction, influenza A, and TNF signaling, were significantly associated with hospitalization in COVID-19 patients (Additional file 2: Table S6 and Additional file 3: Fig. S10).

\section{Integrative analysis of GWAS on severe COVID-19 with GTEx eQTL data}

To obtain combined signals from multiple tissues [58], we leveraged S-MultiXcan to meta-analyze the tissue-specific associations from 49 tissues in GTEx, which showed that the genetically predicted expressions of 16 genes were significantly associated with severe COVID-19 (FDR < 0.05, Fig. $2 \mathrm{C}$ and Additional file 2: Table S7). Of note, 14 of 16 genes (87.5\%) were identified to be significant in MAGMA analysis (Additional file 3: Fig. S11A-B). Through conducting S-PrediXcan analysis of blood and lung tissues that were linked with SARS-CoV-2 infection, we found eight genes whose genetically regulated expression were significantly associated with severe COVID-19 (FDR $<0.05$, Additional file 2: Table S8). Using in silico permutation analysis, we further observed that there existed a high consistence among results from MAGMA, S-PrediXcan, and S-MultiXcan analyses $\left(P<1.0 \times 10^{-5}\right.$, Additional file 3: Fig. S12A-C). The aforementioned multiple genomic analyses identified 34 risk genes that showed supportive evidence of involvement in the etiology of COVID-19 (Additional file 3: Fig. S13A-B).

\section{Functional characterization of $\mathbf{3 4}$ risk genes for severe COVID-19}

The result of a network-based enrichment analysis suggested that 22 of 34 risk genes were significantly enriched in a PPI subnetwork $\left(P=2.85 \times 10^{-13}\right.$, Fig. $\left.2 \mathrm{D}\right)$, which is consistent with the consensus that disease-related genes are more densely connected [59,60]. To functionally characterize the drug targets of these genes, we conducted a drug-gene interaction analysis and identified 11 genes including CCR1, IFNAR2, IL1ORB, and OAS1 

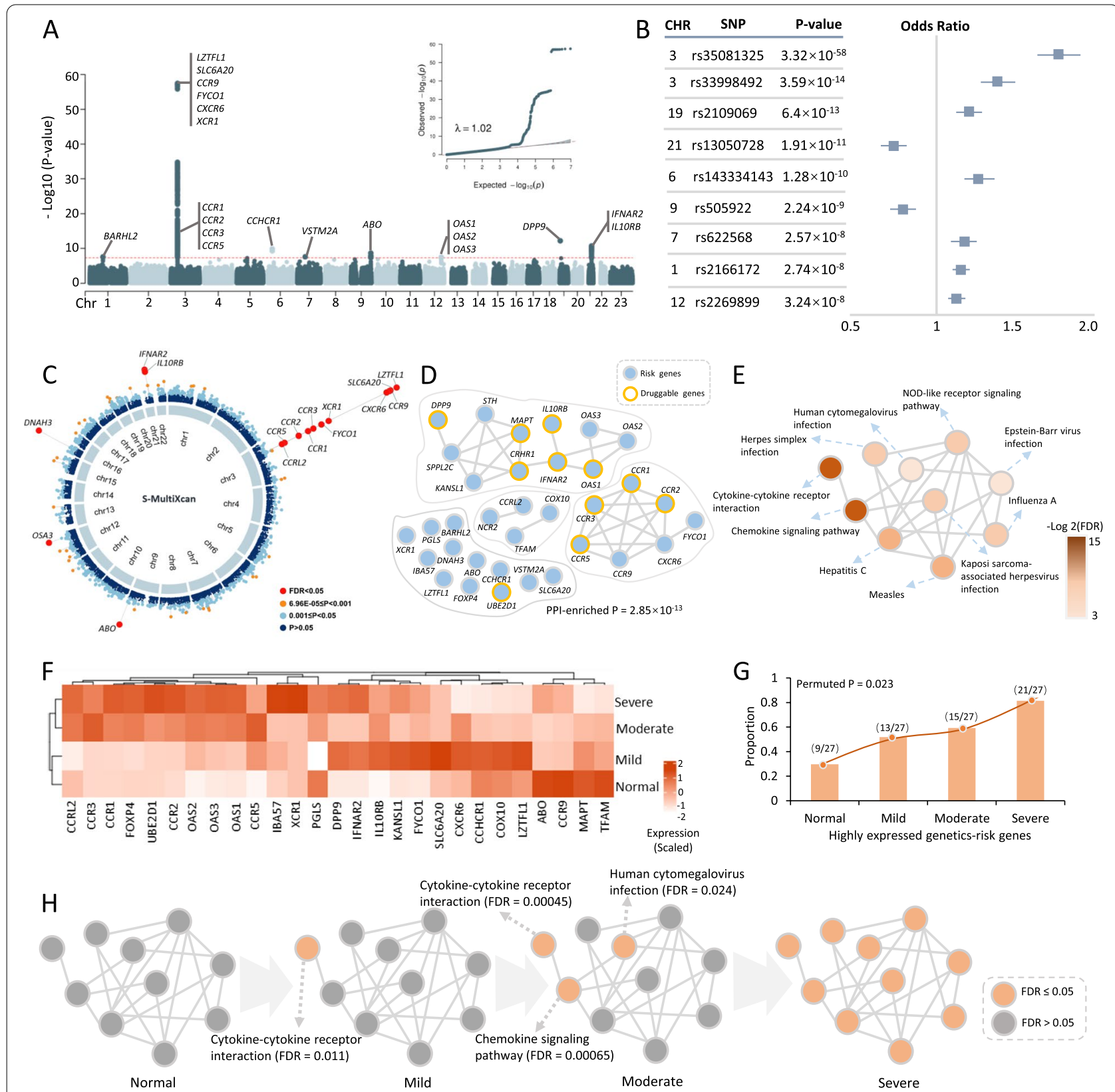

Fig. 2 Risk genes and pathways associated with hospitalized COVID-19 from meta-GWAS summary data. A Manhattan plot and quantile-quantile (QQ) plot of meta-GWAS analysis highlighting eight risk genetic loci for hospitalized COVID-19. The red horizontal line represents the genome-wide significance threshold of $P<5 \times 10^{-8}$. The genomic inflation factor $\lambda=1.02$. B Nine index SNPs within eight genomic loci associated with hospitalized COVID-19. The left panel shows the $P$ value of each index SNP, and the right panel shows the odds ratio with $95 \%$ confidence interval. C Circus plot showing the results of the S-MultiXcan-based analysis. The inner ring demonstrates the 22 autosomal chromosomes (Chr1-22). In the outer ring, a circular symbol represents a specific gene and color marks the statistical significance of the gene for hospitalized COVID-19 (red marks FDR $<0.05$, orange indicates $6.96 \times 10^{-5} \leq P<0.001$, light blue marks $0.001 \leq P \leq 0.05$, and dark blue indicates $P>0.0$ ). D PPI network of these 34 identified risk genes based on the STRING database (v11.0, https://string-db.org/). The orange ring represents druggable genes targeted by at least one known drug. $\mathbf{E}$ Network module constructed by using the Jaccard distance showing the connectivity of 10 significant pathways enriched by 34 risk genes. F Heatmap showing the results of hierarchical clustering analysis of 27 risk genes on COVID-19 severity. Seven risk genes did not express in dataset \#1, and the expression level of each gene was scaled. G The proportion of highly expressed genes among 27 risk genes in normal controls and in the three phases of COVID-19 (mild, moderate, and severe patients). Using 10,000 times of permutation analysis to calculate the significance of the observation (permuted $P=0.023$ ). $\mathbf{H}$ Plot showing an increase of the significantly enriched pathways in the network module with elevated COVID-19 severities. Orange color represents a significantly enriched pathway (FDR $\leq 0.05)$ and gray color represents a non-significantly enriched pathway $(F D R>0.05)$ 
were targeted by at least one known drug (Fig. 2D and Additional file 3: Fig. S14), of which some genes including CCR1, IFNAR2, and IL1ORB have been reported to be drug targets for treating severe COVID-19 patients $[25,26]$. Furthermore, these 34 genes were significantly enriched in a functional module consisting of 10 biological pathways (Fig. 2E, Additional file 2: Table S9 and Additional file 3: Fig. S15), among which two top-ranked ones being cytokine-cytokine receptor interaction and chemokine signaling pathway (FDR $<0.05)$. Most of these enriched pathways have been reported to be implicated in COVID-19 [25, 61, 62].

Based on the expression profile of dataset \#1, we conducted a hierarchical clustering analysis of these identified risk genes on COVID-19 severity and found that these risk genes predisposed to be highly expressed in severe patients compared to the normal group (permuted $P=0.023$, Fig. 2F, G). Consistently, the number of significantly enriched pathways was elevated with increased severities (Fig. 2H). Genes in both cytokine-cytokine receptor interaction and chemokine signaling pathways showed significantly high expressions in the early phase of SARS-CoV-2 infection (Fig. 2H), suggesting that these two pathways could play critical roles in the initiation of COVID-19.

\section{Genetics-influenced peripheral immune cell types for severe COVID-19}

To identify genome-wide host genetic components that have effects on peripheral immune cells for severe COVID-19, we first leveraged a regression-based polygenic model [49] to integrate GWAS summary data on severe COVID-19 with single-cell transcriptomic profiles (dataset \#1) according to different COVID19 severities (see the "Methods" section). We found that CD16+monocytes were significantly associated with three phases of COVID-19, mature B cells showed remarkable associations with mild COVID-19, megakaryocytes were significantly associated with moderate and severe COVID-19, and memory CD8+T cells showed significant associations with severe COVID-19 (permuted $P<0.05$, Fig. 3A). Furthermore, we used a generalized linear regression model [63] to validate these severe COVID-19-associated cell types by conditioning on the $10 \%$ most specific genes for each type and consistently found that CD16+monocytes and megakaryocytes showed notable associations with severe COVID-19 $(P<$ 0.05 , see Additional file 1: Supplementary methods). We found that $\mathrm{CD} 16+$ monocytes tended to be associated with severe COVID-19 among patients with younger age, female, and low BMI, whereas memory CD8+ T cells predisposed to be associated with severe COVID19 among patients with elder age, male, and high BMI.
Smoking behaviors contribute a higher risk to the association of both CD16+ monocytes and memory CD8+ $\mathrm{T}$ cells with severe COVID-19 (Additional file 3: Fig. S16-S19). Additionally, using the Cell-ID method [64], we found that 34 GWAS-identified genes score of individual cells were higher detected in $\mathrm{CD} 16+$ monocytes and memory CD8+T cells (Additional file 3: Fig. S20). These results indicated that $\mathrm{CD} 16+$ monocytes, megakaryocytes, and memory CD8+T cells were more vulnerable to the influence of genetic components on severe-stage patients.

Based on the specificity algorithm used in MAGMA [63], we noticed that the top specific cell type of CCR1 was CD16+monocytes, CXCR6 was most specifically expressed in memory $\mathrm{CD} 8+\mathrm{T}$ cells, and $A B O$ was specific to megakaryocytes (Additional file 3: Fig. S21A), recalling that CXCR6, CCR1, and $A B O$ were prioritized to be candidate causal genes for severe COVID-19 based on the V2G score in above genetics-based analysis. Compared with other cell types, CCR1 was primarily expressed in CD16+monocytes (24.77\%), CXCR6 was mainly expressed in memory CD8+T cells $(40.29 \%)$, and the $A B O$-expressed cells were highly specific to megakaryocytes (54.63\%) (Additional file 2: Table S10 and Additional file 3: Fig. S21B). To gather additional empirical support, we analyzed the combined dataset of both datasets \#2 and \#3 as a validation and found CCR1, CXCR6, and $A B O$ showed a consistent specificity in the three cell types (Additional file 3: Fig. S22).

Given that the primary goal of the current study was to characterize genetic factors that exert an effect on peripheral immune cell types for severe COVID-19, the majority of our subsequent detailed analyses would be concentrated on three immune cell subpopulations: $C C R 1^{+} \mathrm{CD} 16+$ monocytes, $A B O^{+}$megakaryocytes, and $\mathrm{CXCR6}^{+}$memory CD8+T cells (Fig. 3B).

\section{$\mathrm{CCR} 1+\mathrm{CD} 16+$ monocytes and $\mathrm{ABO}^{+}$megakaryocytes exacerbating inflammation in severe COVID-19}

The accumulating lines of evidence [12,65] have suggested that subsets of monocytes and megakaryocytes might be the major resources of aggressive hyper-inflammatory response (named as cytokine storm) [52]. We sought to examine whether $C C R 1^{+} \mathrm{CD} 16+$ monocytes and $\mathrm{ABO}^{+}$megakaryocytes play more important roles in cytokine storm among severe patients. As for $C C R 1^{+} \mathrm{CD} 16+$ monocytes, we found that the inflammatory cytokine score was significantly higher than that of CCR1- CD16+monocytes $\left(P=2.5 \times 10^{-7}\right.$, Fig. 4A, Additional file 2: Table S11 and Additional file 3: Fig. S24A). Consistently, the combined score of both cytokine-cytokine receptor interaction and chemokine signaling pathway was prominently higher in $C C R 1^{+}$ 


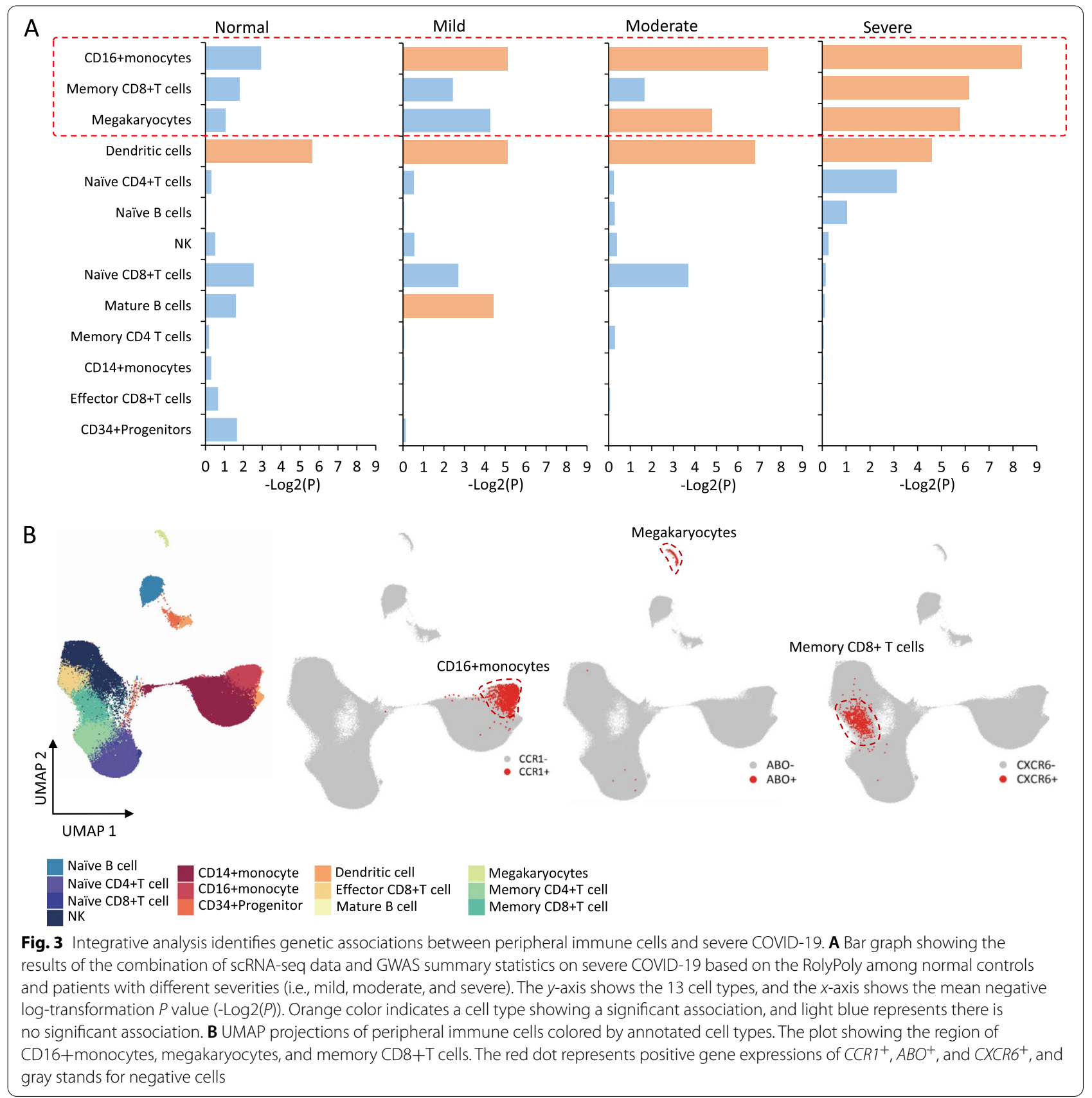

CD16+monocytes $\left(P<2.2 \times 10^{-16}\right.$, Additional file 3 : Fig. 23A and S24B). These results were validated by using the independent method of Cell-ID [64] (Additional file 1: Supplementary methods and Additional file 3: Fig. S29). Compared with $C C R 1^{-} \mathrm{CD} 16+$ monocytes, there were 351 significantly highly expressed genes in $C C R 1^{+}$ CD16+monocytes, such as inflammatory and cytokine genes of IL1B, IL27, CXCL10, CXCL8, CD14, and OSM (FDR < 0.05, Fig. 4B and Additional file 2: Table S12), which have been documented to be associated with the inflammatory response and chemotaxis of immune cells among COVID-19 patients [10, 15, 66, 67]. Functionally, 19 KEGG pathways were significantly overrepresented by the 351 highly expressed genes (FDR < 0.05, Fig. 4C and Additional file 2: Table S13), including cytokine-cytokine receptor interaction and chemokine signaling pathway, reminiscing that most of them were identified in above genetics-based pathway analysis. Additionally, these highly expressed genes among $C C R 1^{+} \mathrm{CD} 16+$ monocytes have a remarkably higher 

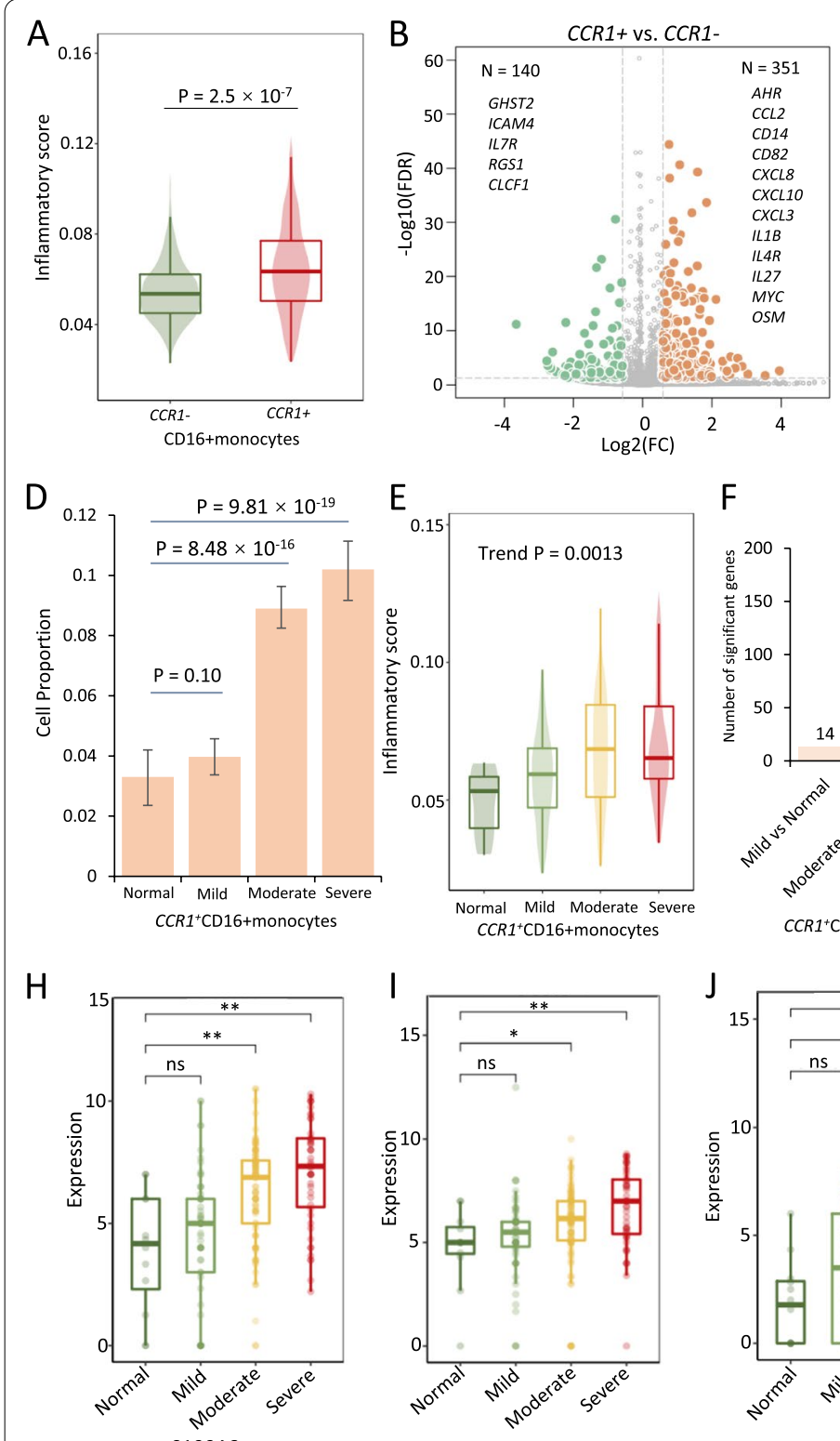

S100A9

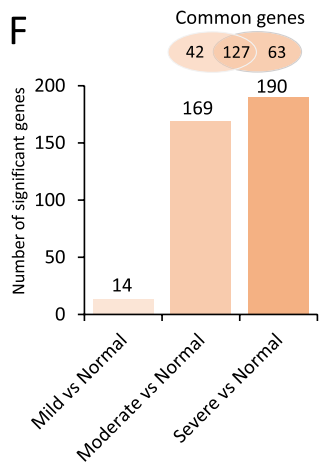

CCR1 ${ }^{+} \mathrm{CD} 16+$ monocytes

C
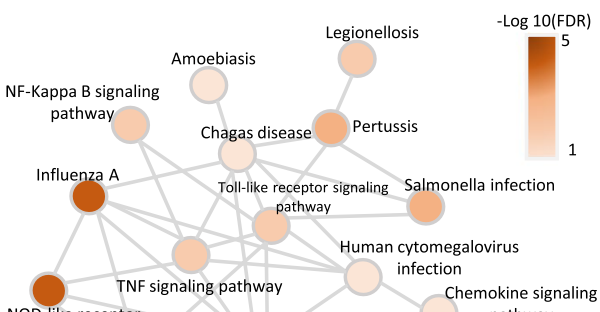
NOD-like receptor
signaling pathway

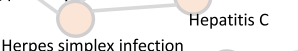
Chemokine signaling
pathway

Herpes simplex infection Staphylococcus aureus infection

CCR1+CD16+monocytes

\section{G}
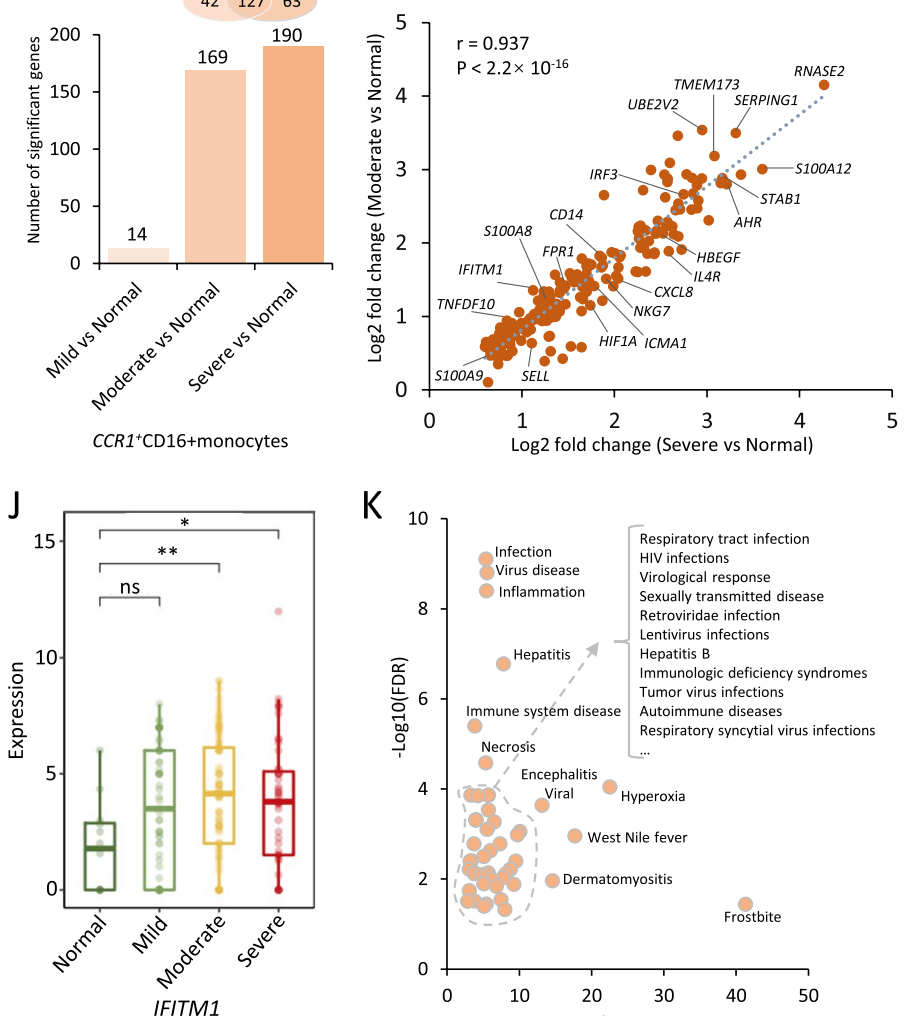

K

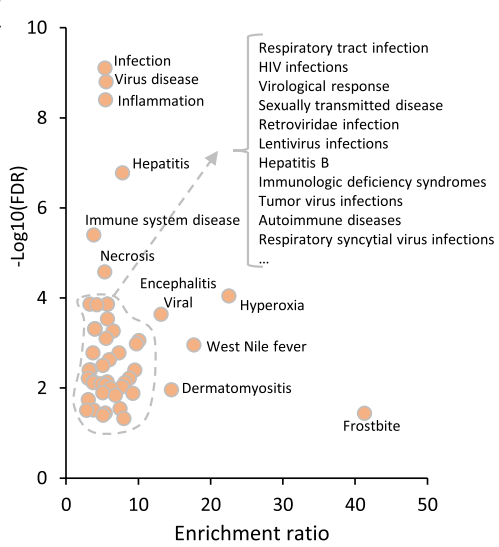

Fig. $4 \mathrm{CCR} 1^{+} \mathrm{CD} 16+$ momocytes contribute higher risk to cytokine storms among severe COVID-19 patients. A Boxplot showing the difference in inflammatory cytokine score between $C C R 1^{+}$and $C C R 1^{-} C D 16+$ monocytes. A two-side Wilcoxon sum-rank test was used. B Volcano plot showing differentially expressed genes between $C C R 1^{+}$and $C C R 1^{-} C D 16+$ monocytes. $C$ Significantly enriched pathways by 351 highly expressed genes among $C C R 1^{+} \mathrm{CD} 16+$ monocytes. Color legend represents the log-transformed FDR value (-Log10(FDR)). D Bar graph showing the proportion of $C C R 1^{+} C D 16+$ monocytes among normal, mild, moderate, and severe groups. E Boxplot showing the inflammatory Cytokine sCore of $C C R 1^{+}$ CD16+ monocytes among normal, mild, moderate, and severe groups. The Mann-Kendall trend analysis was used. F Bar graph showing the differentially up-DEGs among different COVID-19 patients compared with normal controls. Namely, mild COVID-19 vs. normal, moderate COVID-19 vs. normal, and severe COVID-19 vs. normal. Venn plot on top of bar showing the overlapped up-DEGs between moderate and severe patients. G The correlation of up-DEGs between moderate and severe patients. Pearson correlation analysis was used to calculate the correlation coefficient and $P$ value. H-J Representative up-DEGs among $C C R 1^{+}$CD16+ monocytes showing significantly elevated expressions with increased COVID-19 severities. HS100A8, IS100A9, and JIFITM1. K Disease-term enrichment analysis on 190 up-DEGs based on the GLAD4U database. The $y$-axis shows -Log10(FDR), and the $x$-axis shows the enrichment ratio 
proportion of druggable genes and COVID-19-associated druggable genes $(P \leq 0.01$, Additional file 2 : Table S14 and Additional file 3: Fig. S23B).

The cell percentage of $\mathrm{CCR}^{+} \mathrm{CD} 16+$ monocytes showed a notable elevation among moderate and severe patients compared with normal controls $(P<0.001)$, with no significant difference between mild patients and normal controls ( $P=0.1$, Fig. 4D). Furthermore, the inflammatory cytokine scores among $C C R 1^{+} \mathrm{CD} 16+$ monocytes were significantly elevated with increased severities (Trend $P=0.0013$, Fig. 4E). In comparison with normal controls, mild, moderate, and severe patients displayed significantly up-regulated expressions (up-DEGs) with 14, 169, and 190 genes respectively (FDR $<0.05$, Fig. 4F and Additional file 3: Fig. S23D). Notably, there existed a high correlation between up-DEGs of moderate and severe patients $\left(r=0.937, P<2.2 \times 10^{-16}\right.$; Fig. 4G), such as S100A8, S100A9, and IFITM1 (Fig. 4H-J), indicating a similar expression pattern between moderate and severe patients. Accumulating release of massive amounts of calprotectin (S100A8/S100A9) in monocytes contributes to inflammatory response among severe COVID-19 patients $[10,12,16]$.

Furthermore, these 190 up-DEGs were significantly enriched in disease terms associated with viral infection and inflammation and 17 functional GO-terms (FDR $<0.05$, Fig. $4 \mathrm{~K}$, Additional file 2: Tables S15-S16 and Additional file 3: Fig. S23E), including interferon alpha/ beta signaling and interferon gamma signaling. These interferon-related genes, including IRF3, IRF2, IFI6, IFITM1, ISG15, and ICAM1, may induce autoinflammatory and autoimmune conditions contributing to the innate immune cells against SARS-CoV-2 infection [68, 69]. Of note, a high proportion of $63.68 \%$ among 190 upDEGs, such as CXCL8, IFITM1, S100A8, and S100A9, were annotated into 15 potential druggable gene categories (Additional file 2: Table S17 and Additional file 3: Fig. S23F-L). These results indicate that interferon-related genes among $C C R 1^{+} \mathrm{CD} 16+$ monocytes have instrumental effects in exacerbating inflammation among severe patients.

In addition, we found that $A B O^{+}$megakaryocytes had a significantly higher inflammatory cytokine score than that in $A B O^{-}$cells by using two independent methods of AddModuleScore in Seurat [30] and Cell-ID [64] $(P<$ 0.001, Additional file 3: Fig. S25A-B, S26A-B and S29). Compared with $A B O^{-}$megakaryocytes, 424 genes were significantly highly expressed in $A B O^{+}$megakaryocytes (FDR $<0.05$, Additional file 2: Table S18 and Additional file 3: Fig. S25C). These 424 highly expressed genes were significantly enriched in systemic lupus erythematosus, alcoholism, and platelet activation (FDR $<0.05$, Additional file 2: Table S19 and Additional file 3: Fig. S25D).
Similar to $C C R 1^{+} \mathrm{CD} 16+$ monocytes, the cell percentage of $\mathrm{ABO}^{+}$megakaryocytes was significantly elevated among moderate and severe patients $(P<0.01$, Additional file 3: Fig. S25E). Among $A B O^{+}$megakaryocytes, 20 and 35 up-DEGs were notably associated with moderate and severe patients, respectively (FDR $<0.05$, Additional file 3: Fig. S25F-G). There was a highly overlapped rate of these up-DEGs between moderate and severe COVID-19 groups, including $A C P 1, S 100 A 8$, and $A 100 A 9$ (18/20 = 90\%, Additional file 3: Fig. S25F-N). These 35 up-DEGs were annotated to 12 druggable gene categories, and significantly enriched in several disease terms, such as shock and thrombocytopenia (Additional file 2: Tables S20-S21 and Additional file 3: Fig. S25H), which were reported to be associated with COVID-19 [70]. Overall, these results suggest that both $C C R 1^{+} \mathrm{CD} 16+$ monocytes and $A B O^{+}$ megakaryocytes contribute higher risk to dysfunctional inflammation among severe patients.

\section{$\mathrm{CXCR6}^{+}$memory CD8+T cells convey risk to severe COVID-19}

Earlier studies $[10,71]$ have indicated that polyfunctional $\mathrm{T}$ cells play important roles in dominating the antiviral infection immune response and can release a substantially higher amount of multiple distinct cytokines and chemokines in comparison to other $\mathrm{T}$ cells. It is plausible to infer that there exist subsets of memory CD8+T cells predisposing to be multi-functional for against SARSCoV-2 infection. We calculated several immunological features to evaluate whether $C X C R 6^{+}$memory $\mathrm{CD} 8+\mathrm{T}$ cells have a higher polyfunctionality than $C X C R 6^{-}$ memory CD8+T cells. Compared with $\mathrm{CXCR6}^{-}$memory $\mathrm{CD} 8+\mathrm{T}$ cells, we found that scores of cytokine, chemokine, IFN- $\mathrm{a} / \beta$ response, $\mathrm{T}$ cell activation, proliferation, and migration were significantly higher among $\mathrm{CXCR6}^{+}$memory CD8+T cells using both AddModuleScore in Seurat [30] and Cell-ID [64] $(P<0.05$, Fig. 5A-D and Additional file 3: Fig. S27A-C, S28A-G and S29). There were 158 highly expressed genes among $\mathrm{CXCR6}^{+}$ memory CD8+T cells in comparison with $\mathrm{CXCR6}^{-}$cells (FDR $<0.05$, Fig. 5E). These highly expressed genes were significantly enriched in two biological pathways of cytokine-cytokine receptor interaction and inflammatory bowel (FDR $<0.05$, Additional file 2: Table S22 and Additional file 3: Fig. S27D). The chemokine signaling pathway showed a suggestive enrichment $(P<0.05)$. These highly expressed genes contained numerous proinflammatory cytokine and chemokine genes, such as CCR1, CCR2, CCR5, CCR6, CCL3L1, IFNGR1, IL18R1, IL23R, MYC, and TNFSF14, which may be associated with the activation of memory CD8+T cells.

Furthermore, the cell proportion of $\mathrm{CXCR6}^{+}$memory $\mathrm{CD} 8+\mathrm{T}$ cells was significantly higher among both mild 


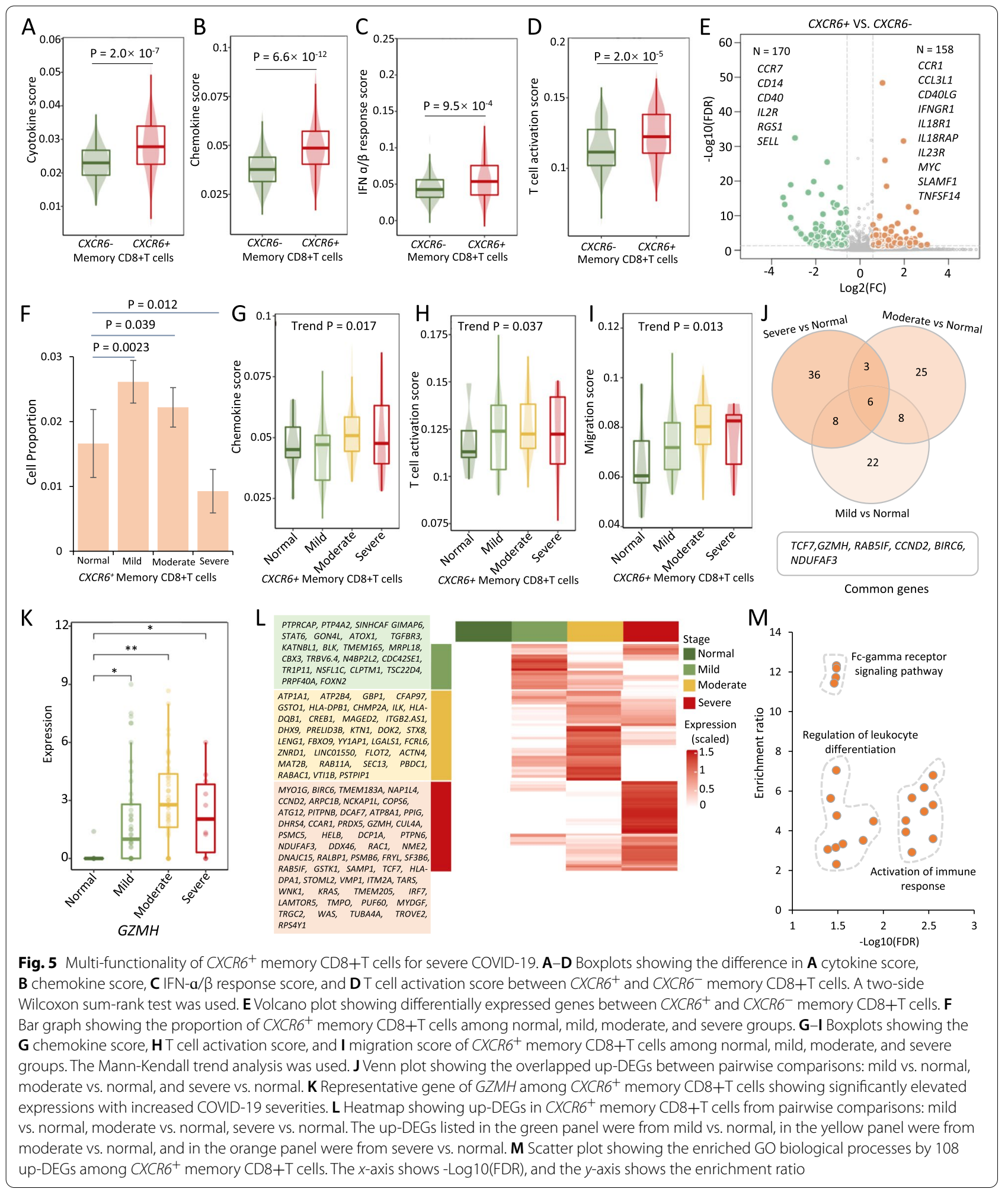

and moderate COVID-19 than that among the normal group $(P<0.05)$, whereas the cell proportion of $C X C R 6^{+}$ memory CD8+T cells among severe COVID-19 was remarkably lower than that among the normal group ( $P$ $=0.012$, Fig. $5 \mathrm{~F}$ ). Consistently, we found that the scores of chemokine, $\mathrm{T}$ cell activation, and migration were 
increased with the increasing patient severities among $\mathrm{CXCR}^{+}$memory CD8+T cells (Trend $P<0.05$, Fig. 5GI) and that lower cytotoxicity score and exhaustion score were observed among moderate-to-severe patients (Trend $P<0.05$, Additional file 3: Fig. S27E-F). Additionally, we found 44,42 , and 53 up-DEGs that were notably associated with mild, moderate, and severe COVID-19, and there were six significant common genes across three phases of COVID-19, including TCF7, GZMH, RAB5IF, CCND2, BIRC6, and NDUFAF3 (Fig. 5J-K and Additional file 3: Fig. S27G-N). The gene of TCF7 was an essential factor in memory $\mathrm{CD} 8+\mathrm{T}$ cell differentiation [72], and $G Z M H$ was reported to mediate antiviral activity through direct cleavage of viral substrates [73]. These 108 up-DEGs were found to be significantly enriched in 22 functional GO-terms, including Fc-gamma receptor signaling pathway, regulation of leukocyte differentiation, and activation of immune response (Fig. 5L, M and Additional file 2: Table S23). Overall, these results indicated that $\mathrm{CXCR6}^{+}$memory $\mathrm{CD} 8+\mathrm{T}$ cells have an enhanced propensity to be multi-functional and activated $\mathrm{T}$ cells involved in severe COVID-19.

\section{Elevated interactions of $\mathrm{CXCR}^{+}$memory CD8+T cells with epithelial cells among severe COVID-19}

To gain refined insights into $C C R 1^{+} \mathrm{CD} 16+$ monocytes and $\mathrm{CXCR6}^{+}$memory $\mathrm{CD} 8+\mathrm{T}$ cells, we examined the cellular interactions among cell populations in PBMCs and BALFs according to the COVID-19 disease status using the CellChat algorithm [53]. For $C C R 1^{+}$ CD16+monocytes in PBMCs, we found an increase in cell-to-cell interactions with other immune cells among severe patients than that in normal controls $(P$ $<0.05$, Fig. 6A and Additional file 3: Fig. S30). There was no statistical difference in cellular communications of $C C R 1^{-}$CD16+monocytes with other cells between normal and COVID-19 patients $(P>0.05$, Fig. 6B). Compared with normal controls, $C C R 1^{+}$ CD16+monocytes showed elevated interactions with megakaryocytes, memory CD8+T cells, NK, effector CD8+T cells, and CD14+monocytes among severe patients (Additional file 3: Fig. S30). There were 14 ligand-receptor interactions remarkably dominated among severe patients (Fig. $6 \mathrm{C}$ and Additional file 3: Fig. S31A), including ANXA1-FPR1, ITGB2-ICAM2/ CD226, LGALS9-CD44, SELPLG-SELL/SELP, APPCD74, and THBS1-CD36/CD47.

With regard to $\mathrm{CXCR6}^{+}$memory $\mathrm{CD} 8+\mathrm{T}$ cells in PBMCs, the predicted cell-to-cell interactions showed an elevation with increased severities of COVID-19 ( $P$ $<0.05$, Fig. 6D). Similar to $C C R 1^{-}$CD16+monocytes, we observed no remarked difference of cellular interactions between normal controls and COVID-19 patients among $C X C R 6^{-}$memory CD8+T cells $(P>0.05$, Fig. 6E). Compared with healthy individuals, $C X C R 6^{+}$memory CD8+T cells demonstrated higher cellular communications with CD14+monocytes, CD34+progenitors, dendritic cells, effector CD8+T cells, naïve CD8+T cells, memory CD4+T cell, naïve CD4+T cells, NK, and megakaryocytes among severe patients (Additional file 3: Fig. S30). There were 20 elevated cellular interactions of $\mathrm{CXCR6}^{+}$memory CD8+T cells with other immune cells among severe patients, including ANXA1-FPR1, THBS1-CD47, CD99-CD99, ICAM2-(ITGAL+ITGB2), and ITGB2-ICAM2/CD226 (Fig. 6F and Additional file 3: Fig. S31B). These cell adhesion molecules ( $A N X A 1$ and ICMA2), cytokine binding and receptor activity genes (CD44, CD45, CD47, CD74, and THBS1), and inflammatory genes (FPR1 and SELL) have been reported to be associated with COVID-19 [16, 66, 74, 75].

Among BALF cells, we also observed an increase in cellular interactions of $C C R 1^{+} \mathrm{CD} 16+$ monocytes and $\mathrm{CXCR6}^{+}$memory CD8+T cells comparing to their corresponding negative cells $(P<0.001$, Fig. 6G-J and Additional file 3: Fig. S32A), for example, enhanced ligand-receptor axes of SELPLG-SELL, CCL5-CCR1, FN1-(ITGA4+ITGB1), CD99-CD99, and APP-CD74 among $C C R 1^{+}$CD16+monocytes (Fig. 6H), as well as CXCL16-CXCR6, TNFSF14-TNFRSF14, ITGB2-CD226, CLEC2B/CLEC2C-KLRB1, and CCL3/CCL4-CCR5 among $\mathrm{CXCR6}^{+}$memory CD8+T cells (Fig. 6J). Notably, there was a $60 \%$ increase in cellular interactions between $C C R 1^{+}$CD16+monocytes and epithelial cells compared with that of $C C R 1^{-} \mathrm{CD} 16+$ monocytes (Additional file 3: Fig. S32B). We also found a 33.33\% increase in the interactions between $\mathrm{CXCR6}^{+}$memory CD8+T cells and epithelial cells compared with that of $C X C R 6^{-}$ memory CD8+T cells (Additional file 3: Fig. S32C), such as enhanced ligand-receptor interactions including TNF-TNFRSF1A, CXCL16-CXCR6, and CCL3-CCR5. Previous studies $[76,77]$ have reported that the CXCL16CXCR6 axis modulates the localization of tissue-resident memory CD8+T cells to the lung airways. Overall, these results suggest that the increased cellular interactions of $\mathrm{CXCR6}^{+}$memory CD8+T cells with epithelial cells probably enhance the residence of this specific population of $\mathrm{T}$ cells to the lung airways for against SARS-CoV-2 infection.

\section{Discussion}

By using large-scale genetics data, we identified eight genomic loci including three novel loci (e.g., 1p22.2, $6 \mathrm{p} 21.33$, and $7 \mathrm{p} 11.2$ ) that were significantly associated with severe COVID-19. Other five loci including 3p21.31, 9q34.2, 12q24.13, 19p13.3, and 21q22.11 have been reported to be involved in COVID-19 risk in 


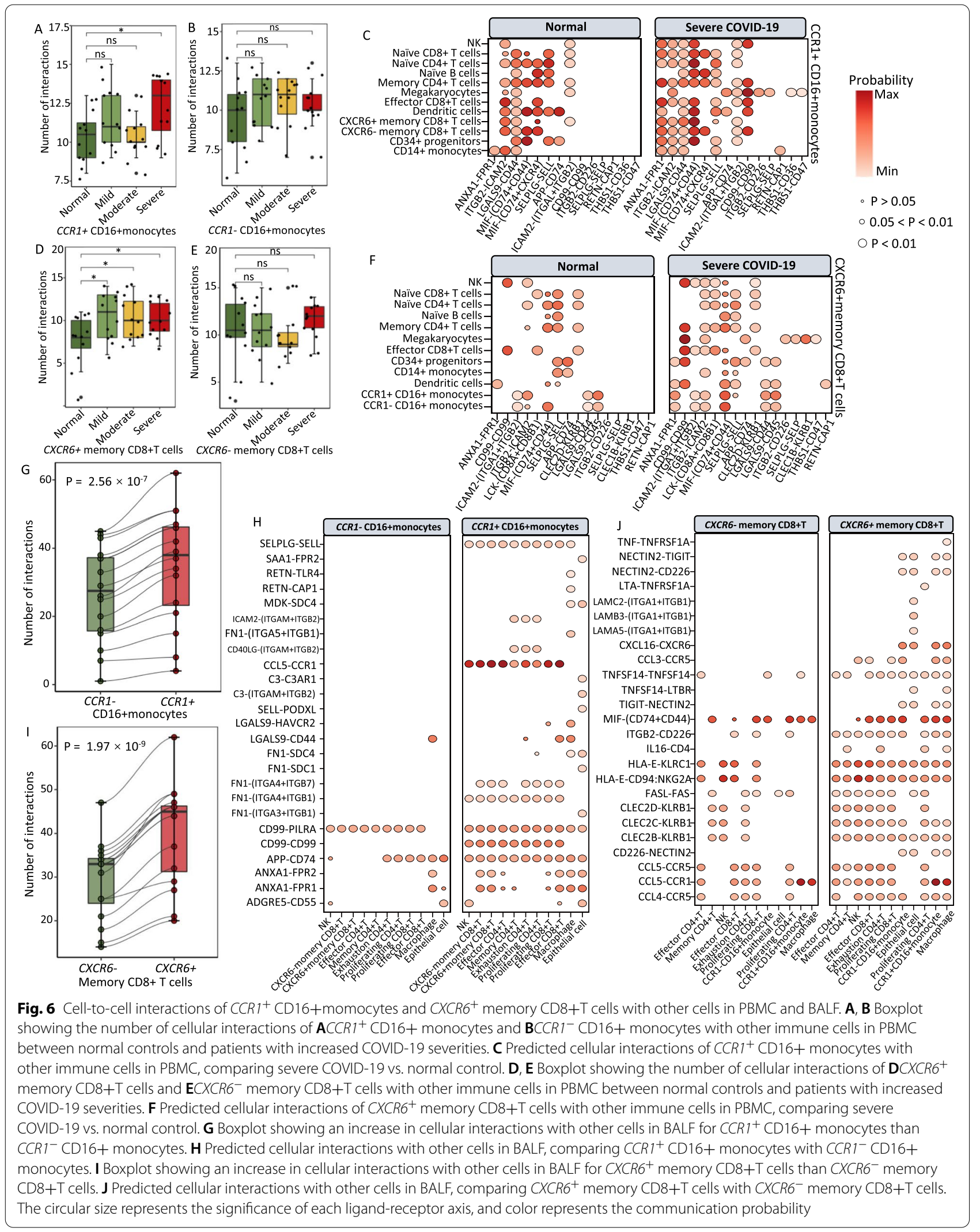


previous studies [23-28]. Notably, we prioritized 34 risk genes, including potential causal genes of CXCR6, CCR1, and $A B O$, to be associated with severe COVID-19. The CXC motif chemokine receptor 6 (CXCR6), which is a $\mathrm{G}$ protein-coupled receptor with seven transmembrane domains, regulates the partitioning of resident memory $\mathrm{T}$ cells by recruiting lung tissue-resident memory CD8+T cells to airways [76]. CCR1 gene encodes the CC motif chemokine receptor 1 (CCR1) belonging to a member of the beta chemokine receptor family. Several previous GWASs have reported genetic variants in CCR1 are associated with COVID-19 susceptibility at a genome-wide significant level $[25,27]$. For the $A B O$ gene, it encodes protein relevant to the $\mathrm{ABO}$ blood group system. Both genetic and non-genetic studies [25, 27, 78] have showed the involvement of $A B O$ gene in COVID-19 susceptibility, while the $A B O$ gene encodes protein that is relevant to the ABO blood group system, and it was also notably associated with several thrombotic and coagulation-related traits including deep vein thrombosis and pulmonary heart disease, which have been reported to be risk factors and sequalae to severe COVID-19 [79, 80].

Understanding the immune responses of monocytes and memory $\mathrm{T}$ cells is fundamental to the rational design of innovative and effective strategies to develop better vaccines $[81,82]$ and contributes to reveal the pathogenesis of severe COVID-19 [12]. Our current analyses reveal that host genetic determinants have a prominent influence on the immune responses of CD16+monocytes, megakaryocytes, and memory CD8+T cells to severe COVID-19. Previous studies [11, $12,65]$ showed that the influence caused by monocytes and megakaryocytes in inflammatory storms is noteworthy among severe COVID-19 patients. We found that $C C R 1^{+} \mathrm{CD} 16+$ monocytes and $\mathrm{ABO}^{+}$megakaryocytes showed a significantly increased propensity to cause inflammatory storms among severe patients. The observations suggest highly expressed interferon-related genes among the two cell subsets, including S100A8, S100A9, S100A12, CD14, CXCL8, IGSF6, IRF3, IFI6, IFITM1, and IFITM3, contribute to exacerbate inflammation among severe patients. The inflammatory mediator of ENRAGE encoded by $S 100 A 12$ was significantly correlated with COVID-19 [21], and S100A8, S100A9, IRF3, IFI6, IFITM1, and IFITM3 have been reported to elicit autoinflammatory and autoimmune conditions in response to SARS-CoV-2 infection [10, 12, 16, 68, 69]. Double-positive CD14+CD16+monocytes reported as tissue-infiltrative cells have a higher potency of antigen presentation and highly expressed proinflammatory cytokines [83, 84]. Additionally, interferons are the mediators in several canonical host antiviral signaling for activating the expression of numerous required molecules of the early response to viral infection [85], and impaired type I interferon activity plays important roles in severe COVID-19 [69]. Our findings described above suggest that $C C R 1^{+}$ $\mathrm{CD} 16+$ monocytes and $\mathrm{ABO}^{+}$megakaryocytes as two functional subsets of myeloid cells convey higher risks to severe COVID-19.

Memory CD8+T cells could elicit immunization that shows enhanced functional features contributing to protect host from viral infectious [82]. After influenza virus infections, memory $\mathrm{CD} 8+\mathrm{T}$ cells reside in the lung for a couple of months and these resident memory $\mathrm{T}$ cells are necessary for effective immunity against secondary infection [86]. Among severe COVID-19 patients, we found that $\mathrm{CXCR}^{+}$memory $\mathrm{CD} 8+\mathrm{T}$ cells undertook several enhanced functional features, including higher scores of cytokine, chemokine, $\mathrm{T}$ cell activation, proliferation, and migration, which suggests $C X C R 6^{+}$memory $\mathrm{CD} 8+\mathrm{T}$ cells potentially contributing to the protection of SARS$\mathrm{CoV}-2$ infection. Among these positive $\mathrm{CXCR6}^{+}$cells, numerous highly expressed cytokine and chemokine genes, including CCR1, CCR2, IFNGR1, and $M Y C$, may work on activating memory $\mathrm{T}$ cells. Earlier evidence indicated that MYC was rapidly but temporally induced during the early stage of $\mathrm{T}$ cell activation [87]. The CCR1 plays a pivotal role in the recruitment of effector immune cells to the site of inflammation, and the pharmacologic inhibition of this gene may suppress immune hyper-activation in severe COVID-19 [15]. Memory CD8+T cells obtained the capability of transforming to effector cells by sensing inflammation from monocytes [82]. Thus, inflammatory $C C R 1^{+} \mathrm{CD} 16+$ monocytes among severe COVID-19 patients potentially accelerate the activation of memory CD8+T cells.

Additionally, we observed a decrease of the cell proportion of $\mathrm{CXCR6}^{+}$memory CD8+T cells among severe patients. This decrease in peripheral blood among severe patients is probably due to efflux to the site of virally infected lung tissue in answer to ongoing tissue damage. Earlier studies $[12,88]$ have reported that functional $\mathrm{CD} 8+\mathrm{T}$ cell subsets manifest a notable decrease in the peripheral blood of severe COVID-19 patients. The epithelium is the most vulnerable tissue to be attacked by viral or microbial infection; thus, the presence of resident memory CD8+ $\mathrm{T}$ cells is imperative for defending the debilitating infections for hosts [86]. In the current study, we found an increase in cellular interactions of $\mathrm{CXCR6}^{+}$memory CD8+T cells with epitheliums. Enhanced ligand-receptor interactions including TNF-TNFSFRSF1A, CXCL16-CXCR6, and CCL3-CCR5 may contribute to the lung residence of memory CD8+T cells. Previous evidence demonstrated a major role for CXCL16-CXCR6 interactions in regulating the residence of virus-specific memory CD8+T cells [76, 77]. An 
earlier study showed a stronger interaction between epithelial and immune cells among severe COVID-19 cases than that among moderate cases [15]. We demonstrated that $\mathrm{CXCR6}^{+}$memory CD8+T cells mounted highly effective immune responses to against COVID-19, highlighting the remarkable biological plasticity in subsets of memory CD8+T cells differentiation.

The power of this study is limited by the lack of matched genetic data and scRNA-seq data in each sample for uncovering the genetic effects on immune cells for severe COVID-19. To reduce the influence of this limitation, we adopted a widely used approach by integrating a large-scale GWAS summary statistics with an enormous amount of single-cell sequencing data, as referenced in previous studies $[49,89,90]$. As referenced to previous studies [91-94], we excluded the major histocompatibility complex (MHC) region from all genomic analyses to avoid the confounding of methods by the unusual genetic architecture and extensively high levels of LD at this locus, which could lead to the inflation of identified COVID-19-associated genes and pathways. However, it should be noticed that there might exist unidentified risk genes implicated in severe COVID-19 in this region. Based on our findings suggesting that host genetic components exert regulatory effects on immunological dysregulations for SRAS-CoV-2 infection, more studies are warranted for exploring the genetic modification of peripheral $\mathrm{T}$ cells to defend against lethal severe COVID-19.

\section{Conclusions}

In sum, we provide comprehensive insights that host genetic determinants are fundamental in influencing the peripheral immune responses to severe COVID-19. Both $C C R 1^{+} \mathrm{CD} 16+$ monocytes and $\mathrm{ABO}^{+}$megakaryocytes contribute higher risk to the dysfunctional inflammatory response among severe patients. $\mathrm{CXCR6^{+ }}$ memory CD8+T cells exhibit a notable polyfunctionality including high expression of cytokines and chemokines, as well as enhanced activation and proliferation of $\mathrm{T}$ cells in severe COVID-19 patients. Further experiments to parse the molecular mechanism of three cell subpopulations are crucial for understanding the inter-individual variation of the initiation and progression of COVID-19.

\footnotetext{
Abbreviations

COVID-19: Coronavirus disease 2019; SARS-CoV-2: Severe acute respiratory syndrome coronavirus 2; GWAS: Genome-wide association study; scRNA-seq: Single-cell RNA sequencing; PBMCs: Peripheral blood mononuclear cells; BALF: Bronchoalveolar lavage fluid; eQTL: Expression quantitative trait loci; GEO: The Gene Expression Omnibus database; WHO: The World Health Organization; SNP: Single nucleotide polymorphism; OR: Odds ratio; MAF: Minor allele frequency; QQ: Quantile-quantile; MAGMA: Multi-marker Analysis of GenoMic Annotation; LD: Linkage disequilibrium; FDR: False discovery rate; KEGG: The Kyoto Encyclopedia of Genes and Genomes; PPC: The Pearson correlation
}

coefficient; MDS: Multidimensional scaling; OTG: The Open Target Genetics; PPI: Protein-protein interaction; up-DEG: Significantly up-regulated expression gene associated with COVID-19.

\section{Supplementary Information}

The online version contains supplementary material available at https://doi. org/10.1186/s13073-022-01021-1.

Additional file 1. Supplementary methods

Additional file 2: Table S1. Samples collected from four independent scRNA-seq datasets on COVID-19. Table S2. Selected well-known markers used to define cell types in PBMCs. Table S3. Significant SNPs associated with severe COVID-19 identified by meta-GWAS analysis. Table S4. Replication of these identified loci by using samples with very severe respiratory confirmed COVID-19. Table S5. Significant genes associated with severe COVID-19 identified by MAGMA gene-based association analysis. Table S6. Significant enriched pathways associated with severe COVID-19 identified from MAGMA-based pathway enrichment analysis. Table S7. The 16 significant genes associated with severe COVID-19 identified by S-MultiXcan analysis based on 49 tissues from GTEx consortium. Table S8. The eight significant genes associated with severe COVID-19 identified by S-PrediXcan analysis based on lung and blood tissues. Table S9. The biological pathways enriched by 34 risk genes associated with severe COVID-19. Table S10. The percentage of three severe COVID-19-risk genes expressed in all 13 distinct cell types in PBMCs. Table S11. Summary of inflammatory and cytokine-related genes and genes in two identified KEGG pathways. Table S12. Highly-expressed inflammatory and cytokine genes among CCR1+CD16+monocytes. Table S13. Pathway enrichment analysis of 351 highly-expressed genes among CCR1+CD16+monocytes. Table S14. Druggble proteins collected from the ChEMBL database. Table S15. Functional enrichment analysis of 190 up-DEGs associated with severe COVID-19 based on the Reactome database. Table S16. Disease-based enrichment analysis of 190 up-DEGs associated with severe COVID-19 among CCR1+ CD16+monocytes based on the GLAD4U database. Table S17. 190 up-DEGs associated with severe COVID-19 among CCR1+CD16+monocytes matched in druggable gene categories based on the DGIdb resource. Table S18. Highly-expressed inflammatory and cytokine genes among $A B O+$ megakaryocytes. Table S19. Pathway enrichment analysis of 424 highly-expressed genes among $A B O+$ megakaryocytes. Table S20. Disease-term enrichment analysis of 35 up-DEGs associated with severe COVID-19 among $A B O+$ megakaryocytes based on the GLAD4U database. Table S21. 35 up-DEGs significantly associated with severe COVID-19 among ABO+ megakaryocytes matched in druggable gene categories. Table S22. Pathway enrichment analysis of 158 highly-expressed genes among CXCR6+ memory CD8+T cells. Table S23. GO-terms enrichment analysis of 108 up-DEGs associated with COVID-19 among CXCR6 ${ }^{+}$memory CD8+T cells.

Additional file 3: Fig. S1. UMP projections of cells in PBMCs from normal controls, mild, moderate, and severe COVID-19 patients by using the Seurat R package (dataset \#1). Fig. S2. Heatmap showing levels of well-known marker genes specific for each cell type in PBMCs. Fig. S3. Single-cell transcriptomes of PBMCs from normal controls, mild, moderate, and severe COVID-19 patients. Fig. S4. Hierarchical clustering using the PCC of a normalized transcriptome between controls and patients in cell type resolution. Fig. S5. Boxplots showing percentages of each cell type for PBMCs in donors from healthy control and COVID-19 patients. Fig. S6. scCODA determines the compositional differences of each cell type in PBMCs among donors from healthy control and COVID-19 patients. Fig. S7. Regional association plots for severe COVID-19-associated genetic loci based on meta-GWAS summary data. Fig. S8. Regional association plots for severe COVID-19-associated genetic loci based on meta-GWAS summary data. Fig. S9. Circus plot showing the results of MAGMA-based gene-level association analysis. Fig. S10. The 19 biological pathways enriched from the MAGMA-based pathway enrichment analysis. Fig. S11. High consistence results between MAGMA and S-MultiXcan analysis. Fig. S12. In silico permutation analysis of 100,000 times of random selections. Fig. S13. Multiple independent approaches identify genetics-relevant risk genes associated with severe COVID-19. Fig. S14. Plot of gene-drug 
interaction analysis for 34 risk genes. Fig. S15. The 10 biological pathways significantly enriched by 34 risk genes based on the KEGG database. Fig. S16. Barplots showing the results of RolyPoly among COVID-19 patients stratified by patient's age. Fig. S17. Barplots showing the results of RolyPoly among COVID-19 patients stratified by patient's sex. Fig. S18. Barplots showing the results of RolyPoly among COVID-19 patients stratified by patient's BMI. Fig. S19. Barplots showing the results of RolyPoly COVID-19 patients stratified by patient's smoking status. Fig. S20. CellID-based enrichment in GWAS-identified gene signatures (34 genes) of CD16+monocytes and memory CD8+ T cells using scRNA-seq dataset. Fig. S21. Genetics-risk genes influenced three immune cell subsets for severe COVID-19. Fig. S22. Dot plot showing the expressed percent of three risk genes of $C X C R 6, C C R 1$, and $A B O$ in each peripheral cell type in PBMCs among severe patients based on two sCRNA-seq dataset of \#2 and \#3. Fig. S23. $C \mathrm{CR} 1^{+} \mathrm{CD} 16+$ monocytes showing higher risk to cytokine storms among COVID-19 patients. Fig. S24. Boxplots showing the difference of inflammatory cytokine score and pathway activation score of CD16+ monocytes among normal controls and COVID-19 groups. Fig. S25. $\mathrm{ABO}^{+}$megakaryocytes contribute higher risk to cytokine storms among severe COVID-19 patients. Fig. S26. Boxplots showing the difference of inflammatory cytokine score and pathway activation score of and megakaryocytes among normal controls and COVID-19 groups. Fig. S27. Evidence showing the multi-functionality of $\mathrm{CXCR}^{+}$memory $\mathrm{CD} 8+\mathrm{T}$ cells for severe COVID-19. Fig. S28. Boxplots showing the different score of several immunological features between $C X C R 6^{+}$and $C X C R \sigma^{-}$memory CD8+T cells among normal controls and COVID-19 patients. Fig. S29. Cell-ID-based enrichment in functional terms of CD16+monocytes, megakaryocyte, and memory CD8+ T cells using scRNA-seq dataset. Fig. S30. Differences in the number of predicated cell-to-cell interactions in PBMCs. Fig. S31. Predicted cellular interaction of both $C C R 1^{+} \mathrm{CD} 16+$ monocytes and $C X C R 6^{+}$memory CD8+T cells with other immune cells in PBMCs. Fig. S32. Prediction of cell-to-cell interactions of cells in BALFs.

\section{Acknowledgements}

We appreciate Prof. Yang Jian from Westlake University for providing helpful suggestions, and our appreciation also goes to all the authors from the COVID-19 Host Genetic Consortium who have deposited and shared GWAS summary data on public databases and goes to the authors who publicly released the scRNA-seq datasets on PBMC and BALF with distinct COVID-19 severities.

\section{Authors' contributions}

J.S. and Y.M. conceived and designed the study. Y.M., F.Q., C.D., J.L., Y.K.H., Y.R.Z. Y.X., Y.G.Z., and Y.H.Y. contributed to the management of data collection. Y.M., F.Q., C.D., Y.K.H., and J.L. conducted bioinformatics analysis and data interpretation. Y.M., J.S., Z.W., and J.Q. wrote the manuscripts. All authors reviewed and approved the final manuscript.

\section{Funding}

This study was funded by the National Natural Science Foundation of China (61871294 to J.S.), the Scientific Research Foundation for Talents of Wenzhou Medical University (KYQD20201001 to Y.M.), and the Science Foundation of Zhejiang Province (LR19C060001 to J.S).

\section{Availability of data and materials}

All the GWAS summary statistics used in this study can be accessed in the official websites (www.covid19hg.org/results) [22]. The GTEx eQTL data (version 8) were downloaded from Zenodo repository (https://zenodo.org/record/ 3518299\#.Xv6Z6igzbgl) [42]. Four scRNA-seq datasets were downloaded from the GEO database (https://www.ncbi.nlm.nih.gov/gds/?term=GSE14 9689 [18], https://www.ncbi.n/m.nih.gov/geo/query/acc.cgi?acc=GSE158055 [12], and https://www.ncbi.nlm.nih.gov/gds/?term=GSE150861 [11]) and the ArrayExpress database (https://www.ebi.ac.uk/arrayexpress/experiments/ E-MTAB-9357) [10]. All analyzed codes for hypergenometric analysis [95], hierarchical clustering analysis [18], in silico permutation analysis [25], S-PrediXcan [41], S-MultiXcan [43], MDS [39], MAGMA [35], RolyPoly [49], scCODA [54], Cell-ID [64], and CellChat [53] analysis in the "Methods" section are available in an online GitHub repository at https://github.com/mayunlong89/COVID19_ scRNA [40].

\section{Declarations}

Ethics approval and consent to participate

Not applicable.

\section{Consent for publication}

Not applicable.

\section{Competing interests}

The authors declare that they have no competing interests.

\section{Author details}

${ }^{1}$ Institute of Biomedical Big Data, School of Ophthalmology \& Optometry and Eye Hospital, School of Biomedical Engineering, Wenzhou Medical University, Wenzhou 325027, China. ${ }^{2}$ State Key Laboratory for Diagnosis and Treatment of Infectious Diseases, the First Affiliated Hospital, Collaborative Innovation Center for Diagnosis and Treatment of Infectious Diseases, Zhejiang University School of Medicine, Zhejiang 310003, Hangzhou, China. ${ }^{3}$ Wenzhou Institute, University of Chinese Academy of Sciences, Wenzhou 325011, China. ${ }^{4}$ Department of Cardiology, Affiliated Hangzhou First People's Hospital, Zhejiang University School of Medicine, Hangzhou, China.

Received: 5 August 2021 Accepted: 6 February 2022

Published online: 17 February 2022

\section{References}

1. Dong E, Du H, Gardner L. An interactive web-based dashboard to track COVID-19 in real time. Lancet Infect Dis. 2020;20:533-4.

2. Wu Z, McGoogan JM. Characteristics of and important lessons from the coronavirus disease 2019 (COVID-19) outbreak in China: summary of a report of 72314 cases from the Chinese Center for Disease Control and Prevention. JAMA. 2020;323(13):1239-42.

3. Berlin DA, Gulick RM, Martinez FJ. Severe Covid-19. N Engl J Med. 2020;323(13):1239-42.

4. Richardson S, Hirsch JS, Narasimhan M, Crawford JM, McGinn T, Davidson KW, et al. Presenting characteristics, comorbidities, and outcomes among 5700 patients hospitalized with COVID-19 in the New York City area. JAMA. 2020;323:2052-9.

5. Guan WJ, Ni ZY, Hu Y, Liang WH, Ou CQ, He JX, et al. Clinical characteristics of coronavirus disease 2019 in China. N Engl J Med. 2020;382:1708-20.

6. Xu L, Ma Y, Yuan J, Zhang Y, Wang H, Zhang G, et al. COVID-19 quarantine reveals that behavioral changes have an effect on myopia progression. Ophthalmology. 2021;128(11):1652-4.

7. Pedersen SF, Ho YC. SARS-CoV-2: a storm is raging. J Clin Invest. 2020;130:2202-5.

8. Takahashi T, Ellingson MK, Wong P, Israelow B, Lucas C, Klein J, et al. Sex differences in immune responses that underlie COVID-19 disease outcomes. Nature. 2020;588:315-20.

9. Chen G, Wu D, Guo W, Cao Y, Huang D, Wang H, et al. Clinical and immunological features of severe and moderate coronavirus disease 2019. J Clin Invest. 2020;130:2620-9.

10. Su Y, Chen D, Yuan D, Lausted C, Choi J, Dai CL, et al. Multi-omics resolves a sharp disease-state shift between mild and moderate COVID-19. Cell. 2020;183:1479-95 e1420.

11. Guo C, Li B, Ma H, Wang X, Cai P, Yu Q, et al. Single-cell analysis of two severe COVID-19 patients reveals a monocyte-associated and tocilizumab-responding cytokine storm. Nat Commun. 2020;11:3924.

12. Ren $X$, Wen W, Fan $X$, Hou W, Su B, Cai P, et al. COVID-19 immune features revealed by a large-scale single-cell transcriptome atlas. Cell. 2021;184(7):1895-913 e19.

13. Wen W, Su W, Tang H, Le W, Zhang $X$, Zheng $Y$, et al. Immune cell profiling of COVID-19 patients in the recovery stage by single-cell sequencing. Cell Discov. 2020;6:31.

14. Zhang JY, Wang XM, Xing $X, X u Z$ Z, Zhang C, Song JW, et al. Single-cell landscape of immunological responses in patients with COVID-19. Nat Immunol. 2020;21:1107-18. 
15. Chua RL, Lukassen S, Trump S, Hennig BP, Wendisch D, Pott F, et al. COVID19 severity correlates with airway epithelium-immune cell interactions identified by single-cell analysis. Nat Biotechnol. 2020;38:970-9.

16. Silvin A, Chapuis N, Dunsmore G, Goubet AG, Dubuisson A, Derosa L, et al. Elevated calprotectin and abnormal myeloid cell subsets discriminate severe from mild COVID-19. Cell. 2020;182:1401-18 e1418.

17. Schulte-Schrepping J, Reusch N, Paclik D, Baßler K, Schlickeiser S, Zhang $\mathrm{B}$, et al. Severe COVID-19 is marked by a dysregulated myeloid cell compartment. Cell. 2020;182:1419-40 e1423.

18. Lee JS, Park S, Jeong HW, Ahn JY, Choi SJ, Lee H, et al. Immunophenotyping of COVID-19 and influenza highlights the role of type I interferons in development of severe COVID-19. Sci Immunol. 2020;5:eabd1554.

19. Cao X. COVID-19: immunopathology and its implications for therapy. Nat Rev Immunol. 2020;20:269-70.

20. Del Valle DM, Kim-Schulze S, Huang HH, Beckmann ND, Nirenberg S, Wang B, et al. An inflammatory cytokine signature predicts COVID-19 severity and survival. Nat Med. 2020;26:1636-43.

21. Arunachalam PS, Wimmers F, Mok CKP, Perera R, Scott M, Hagan T, et al. Systems biological assessment of immunity to mild versus severe COVID19 infection in humans. Science. 2020:369:1210-20.

22. COVID-19 Host Genetics Initiative. The COVID-19 Host Genetics Initiative, a global initiative to elucidate the role of host genetic factors in susceptibility and severity of the SARS-CoV-2 virus pandemic. Eur J Hum Genet. 2020;28:715-8.

23. Zhou S, Butler-Laporte G, Nakanishi T, Morrison DR, Afilalo J, Afilalo $M$, et al. A Neanderthal OAS1 isoform protects individuals of European ancestry against COVID-19 susceptibility and severity. Nat Med. 2021;27:659-67.

24. Pairo-Castineira E, Clohisey S, Klaric L, Bretherick AD, Rawlik K, Pasko D, et al. Genetic mechanisms of critical illness in COVID-19. Nature. 2021:591:92-8.

25. Ma Y, Huang $Y$, Zhao $S$, Yao $Y$, Zhang $Y$, Qu J, et al. Integrative genomics analysis reveals a 21 q22.11 locus contributing risk to COVID-19. Hum Mol Genet. 2021;30(13):1247-58.

26. Gaziano L, Giambartolomei C, Pereira AC, Gaulton A, Posner DC, Swanson $S A$, et al. Actionable druggable genome-wide Mendelian randomization identifies repurposing opportunities for COVID-19. Nat Med. 2021;27:668-76.

27. Ellinghaus D, Degenhardt F, Bujanda L, Buti M, Albillos A, Invernizzi P, et al. Genomewide association study of severe Covid-19 with respiratory failure. N Engl J Med. 2020;383:1522-34.

28. COVID-19 Host Genetics Initiative. Mapping the human genetic architecture of COVID-19. Nature. 2021;600(7889):472-7.

29. 10x Genomics. https://www.10xgenomics.com/solutions/single-cell/.

30. Butler A, Hoffman P, Smibert P, Papalexi E, Satija R. Integrating single-cell transcriptomic data across different conditions, technologies, and species. Nat Biotechnol. 2018;36:411-20.

31. Waltman $L$, van Eck NJ. A smart local moving algorithm for large-scale modularity-based community detection. Eur Phys J B. 2013;86:471.

32. Korsunsky I, Millard N, Fan J, Slowikowski K, Zhang F, Wei K, et al. Fast, sensitive and accurate integration of single-cell data with Harmony. Nat Methods. 2019;16:1289-96.

33. Auton A, Brooks LD, Durbin RM, Garrison EP, Kang HM, Korbel JO, et al. A global reference for human genetic variation. Nature. 2015;526:68-74.

34. Pruim RJ, Welch RP, Sanna S, Teslovich TM, Chines PS, Gliedt TP, et al. LocusZoom: regional visualization of genome-wide association scan results. Bioinformatics. 2010;26:2336-7.

35. de Leeuw CA, Mooij JM, Heskes T, Posthuma D. MAGMA: generalized gene-set analysis of GWAS data. PLoS Comput Biol. 2015:11:e1004219.

36. Wang J, Duncan D, Shi Z, Zhang B. WEB-based GEne SeT AnaLysis Toolkit (WebGestalt): update 2013. Nucleic Acids Res. 2013;41:W77-83.

37. Kanehisa M, Goto S. KEGG: Kyoto Encyclopedia of Genes and Genomes. Nucleic Acids Res. 2000;28:27-30.

38. Ma Y, Li J, Xu Y, Wang Y, Yao Y, Liu Q, et al. Identification of 34 genes conferring genetic and pharmacological risk for the comorbidity of schizophrenia and smoking behaviors. Aging (Albany NY). 2020;12:2169-225.

39. Hout MC, Papesh MH, Goldinger SD. Multidimensional scaling. Wiley Interdiscip Rev Cogn Sci. 2013:4:93-103.

40. Ma Y QF, Deng C, Li J, Huang Y, Wu Z, Zhou Y, Zhang Y, Xiong Y, Yao J, Zhong Y, Qu J, Su J. Analyzed codes for the immune response of severe
COVID-19 using scRNA-seq data. 2022. https://github.com/mayun long89/COVID19_scRNA.

41. Barbeira AN, Dickinson SP, Bonazzola R, Zheng J, Wheeler HE, Torres JM, et al. Exploring the phenotypic consequences of tissue specific gene expression variation inferred from GWAS summary statistics. Nat Commun. 2018;9:1825.

42. Barbeira AN, Bonazzola R, Gamazon ER, Liang Y, Park Y, Kim-Hellmuth S, et al. Exploiting the GTEx resources to decipher the mechanisms at GWAS loci. Genome Biol. 2021;22:49.

43. Barbeira AN, Pividori M, Zheng J, Wheeler HE, Nicolae DL, Im HK. Integrating predicted transcriptome from multiple tissues improves association detection. PLoS Genet. 2019;15:e1007889.

44. Ma X, Wang $P, X u G, Y u F, M a Y$. Integrative genomics analysis of various omics data and networks identify risk genes and variants vulnerable to childhood-onset asthma. BMC Med Genet. 2020;13:123.

45. Xu M, Li J, Xiao Z, Lou J, Pan X, Ma Y. Integrative genomics analysis identifies promising SNPs and genes implicated in tuberculosis risk based on multiple omics datasets. Aging (Albany NY). 2020:12:19173-220.

46. von Mering C, Huynen M, Jaeggi D, Schmidt S, Bork P, Snel B. STRING: a database of predicted functional associations between proteins. Nucleic Acids Res. 2003;31:258-61.

47. Szklarczyk D, Santos A, von Mering C, Jensen LJ, Bork P, Kuhn M. STITCH 5: augmenting protein-chemical interaction networks with tissue and affinity data. Nucleic Acids Res. 2016;44:D380-4.

48. Cotto KC, Wagner AH, Feng YY, Kiwala S, Coffman AC, Spies G, et al. DGldb 3.0: a redesign and expansion of the drug-gene interaction database. Nucleic Acids Res. 2018:46:D1068-d1073.

49. Calderon D, Bhaskar A, Knowles DA, Golan D, Raj T, Fu AQ, et al. Inferring relevant cell types for complex traits by using single-cell gene expression. Am J Hum Genet. 2017;101:686-99.

50. Chang CC, Chow CC, Tellier LC, Vattikuti S, Purcell SM, Lee JJ. Secondgeneration PLINK: rising to the challenge of larger and richer datasets. Gigascience. 2015;4:7.

51. Puram SV, Tirosh I, Parikh AS, Patel AP, Yizhak K, Gillespie S, et al. Single-cell transcriptomic analysis of primary and metastatic tumor ecosystems in head and neck cancer. Cell. 2017:171:1611-24 e1624.

52. Fajgenbaum DC, June CH. Cytokine Storm. N Engl J Med. 2020:383:2255-73.

53. Jin S, Guerrero-Juarez CF, Zhang L, Chang I, Ramos R, Kuan CH, et al. Inference and analysis of cell-cell communication using CellChat. Nat Commun. 2021;12:1088.

54. Büttner M, Ostner J, Müller CL, Theis FJ, Schubert B. scCODA is a Bayesian model for compositional single-cell data analysis. Nat Commun. 2021;12:6876.

55. Thomson W, Jabbari S, Taylor A, Arlt W, Smith D. Simultaneous parameter estimation and variable selection via the logit-normal continuous analogue of the spike-and-slab prior. J R Soc Interface. 2019;16:20180572.

56. Aitchison J. The statistical analysis of compositional data. J R Stat Soc B (Methodological). 1982;44:139-60

57. Ghoussaini M, Mountjoy E, Carmona M, Peat G, Schmidt EM, Hercules A, et al. Open Targets Genetics: systematic identification of trait-associated genes using large-scale genetics and functional genomics. Nucleic Acids Res. 2021:49:D1311-d1320.

58. Battle A, Brown CD, Engelhardt BE, Montgomery SB. Genetic effects on gene expression across human tissues. Nature. 2017:550:204-13.

59. Wang Q, Chen R, Cheng F, Wei Q, Ji Y, Yang H, et al. A Bayesian framework that integrates multi-omics data and gene networks predicts risk genes from schizophrenia GWAS data. Nat Neurosci. 2019;22:691-9.

60. Ma Y, Li MD. Establishment of a strong link between smoking and cancer pathogenesis through DNA methylation analysis. Sci Rep. 2017;7:1811.

61. Auwul MR, Rahman MR, Gov E, Shahjaman M, Moni MA. Bioinformatics and machine learning approach identifies potential drug targets and pathways in COVID-19. Brief Bioinform. 2021;22(5):bbab120.

62. More SA, Patil AS, Sakle NS, Mokale SN. Network analysis and molecular mapping for SARS-CoV-2 to reveal drug targets and repurposing of clinically developed drugs. Virology. 2021:555:10-8.

63. Bryois J, Skene NG, Hansen TF, Kogelman LJA, Watson HJ, Liu Z, et al. Genetic identification of cell types underlying brain complex traits yields insights into the etiology of Parkinson's disease. Nat Genet. 2020;52:482-93. 
64. Cortal A, Martignetti L, Six E, Rausell A. Gene signature extraction and cell identity recognition at the single-cell level with Cell-ID. Nat Biotechnol. 2021;39:1095-102.

65. Manne BK, Denorme F, Middleton EA, Portier I, Rowley JW, Stubben C, et al. Platelet gene expression and function in patients with COVID-19. Blood. 2020;136:1317-29.

66. Shaath $\mathrm{H}$, Vishnubalaji R, Elkord E, Alajez NM. Single-cell transcriptome analysis highlights a role for neutrophils and inflammatory macrophages in the pathogenesis of severe COVID-19. Cells. 2020;9:2374.

67. Rydyznski Moderbacher C, Ramirez SI, Dan JM, Grifoni A, Hastie KM, Weiskopf D, et al. Antigen-specific adaptive immunity to SARS-CoV-2 in acute COVID-19 and associations with age and disease severity. Cell. 2020;183:996-1012 e1019.

68. King KR, Aguirre AD, Ye YX, Sun Y, Roh JD, Ng RP Jr, et al. IRF3 and type I interferons fuel a fatal response to myocardial infarction. Nat Med. 2017:23:1481-7.

69. Hadjadj J, Yatim N, Barnabei L, Corneau A, Boussier J, Smith N, et al. Impaired type I interferon activity and inflammatory responses in severe COVID-19 patients. Science. 2020;369:718-24.

70. Lippi G, Plebani M, Henry BM. Thrombocytopenia is associated with severe coronavirus disease 2019 (COVID-19) infections: a meta-analysis. Clin Chim Acta. 2020;506:145-8.

71. Ma C, Cheung AF, Chodon T, Koya RC, Wu Z, Ng C, et al. Multifunctional T-cell analyses to study response and progression in adoptive cell transfer immunotherapy. Cancer Discov. 2013;3:418-29.

72. Akondy RS, Fitch M, Edupuganti S, Yang S, Kissick HT, Li KW, et al. Origin and differentiation of human memory CD8 T cells after vaccination. Nature. 2017;552:362-7.

73. Andrade F, Fellows E, Jenne DE, Rosen A, Young CS. Granzyme H destroys the function of critical adenoviral proteins required for viral DNA replication and granzyme B inhibition. EMBO J. 2007;26:2148-57.

74. Li Y, Hou G, Zhou H, Wang Y, Tun HM, Zhu A, et al. Multi-platform omics analysis reveals molecular signature for COVID-19 pathogenesis, prognosis and drug target discovery. Signal Transduct Target Ther. 2021;6:155.

75. Bruchez A, Sha K, Johnson J, Chen L, Stefani C, McConnell H, et al. MHC class II transactivator CIITA induces cell resistance to Ebola virus and SARS-like coronaviruses. Science. 2020;370:241-7.

76. Wein AN, McMaster SR, Takamura S, Dunbar PR, Cartwright EK, Hayward SL, et al. CXCR6 regulates localization of tissue-resident memory CD8 T cells to the airways. J Exp Med. 2019;216:2748-62.

77. Takamura S, Kato S, Motozono C, Shimaoka T, Ueha S, Matsuo K, et al. Interstitial-resident memory CD8(+)T cells sustain frontline epithelial memory in the lung. J Exp Med. 2019;216:2736-47.

78. Zhao J, Yang Y, Huang H, Li D, Gu D, Lu X, et al. Relationship between the ABO Blood Group and the COVID-19 susceptibility. Clin Infect Dis. 2021:73(2):328-31.

79. Klok FA, Kruip M, van der Meer NJM, Arbous MS, Gommers D, Kant KM, et al. Incidence of thrombotic complications in critically ill ICU patients with COVID-19. Thromb Res. 2020;191:145-7.

80. Grillet F, Behr J, Calame P, Aubry S, Delabrousse E. Acute pulmonary embolism associated with COVID-19 pneumonia detected with pulmonary CT angiography. Radiology. 2020;296:E186-e188.

81. Poran A, Harjanto D, Malloy M, Arieta CM, Rothenberg DA, Lenkala D, et al. Sequence-based prediction of SARS-CoV-2 vaccine targets using a mass spectrometry-based bioinformatics predictor identifies immunogenic $T$ cell epitopes. Genome Med. 2020;12:70.

82. Soudja SM, Ruiz AL, Marie JC, Lauvau G. Inflammatory monocytes activate memory CD8(+) T and innate NK lymphocytes independent of cognate antigen during microbial pathogen invasion. Immunity. 2012;37:549-62.

83. Ziegler-Heitbrock L. The CD14+CD16+ blood monocytes: their role in infection and inflammation. J Leukoc Biol. 2007;81:584-92.

84. Kawanaka N, Yamamura M, Aita T, Morita Y, Okamoto A, Kawashima M, et al. CD14+,CD16+ blood monocytes and joint inflammation in rheumatoid arthritis. Arthritis Rheum. 2002;46:2578-86.

85. Hambleton S, Goodbourn S, Young DF, Dickinson P, Mohamad SM, Valappil M, et al. STAT2 deficiency and susceptibility to viral illness in humans. Proc Natl Acad Sci U S A. 2013;110:3053-8.

86. Samji T, Khanna KM. Understanding memory CD8(+) T cells. Immunol Lett. 2017;185:32-9.
87. Nie Z, Hu G, Wei G, Cui K, Yamane A, Resch W, et al. c-Myc is a universal amplifier of expressed genes in lymphocytes and embryonic stem cells. Cell. 2012:151:68-79.

88. Jouan Y, Guillon A, Gonzalez L, Perez Y, Boisseau C, Ehrmann S, et al. Phenotypical and functional alteration of unconventional T cells in severe COVID-19 patients. J Exp Med. 2020;217:e20200872.

89. Finucane HK, Reshef YA, Anttila V, Slowikowski K, Gusev A, Byrnes A, et al. Heritability enrichment of specifically expressed genes identifies diseaserelevant tissues and cell types. Nat Genet. 2018;50:621-9.

90. Xiang B, Deng C, Qiu F, Li J, Li S, Zhang H, et al. Single cell sequencing analysis identifies genetics-modulated ORMDL3+ cholangiocytes having higher metabolic effects on primary biliary cholangitis. J Nanobiotechnol. 2021;19:406.

91. Lv Y, Huang $Y, X u X$, Wang Z, Yu Y, Ma Y, et al. Integrated multi-omics data analysis identifies a novel genetics-risk gene of IRF4 associated with prognosis of oral cavity cancer. medRxiv. 2021.

92. Dong Z, Ma Y, Zhou H, Shi L, Ye G, Yang L, et al. Integrated genomics analysis highlights important SNPS and genes implicated in moderateto-severe asthma based on GWAS and eQTL datasets. BMC Pulm Med. 2020;20:270.

93. Network and Pathway Analysis Subgroup of Psychiatric Genomics Consortium. Psychiatric genome-wide association study analyses implicate neuronal, immune and histone pathways. Nat Neurosci. 2015;18:199-209.

94. Bulik-Sullivan BK, Loh PR, Finucane HK, Ripke S, Yang J, Patterson N, et al. LD Score regression distinguishes confounding from polygenicity in genome-wide association studies. Nat Genet. 2015;47:291-5.

95. Hahne F, Huber W, Gentleman R, Falcon S. Hypergeometric testing used for gene set enrichment analysis; 2010. p. 207-20.

\section{Publisher's Note}

Springer Nature remains neutral with regard to jurisdictional claims in published maps and institutional affiliations.

Ready to submit your research? Choose BMC and benefit from:

- fast, convenient online submission

- thorough peer review by experienced researchers in your field

- rapid publication on acceptance

- support for research data, including large and complex data types

- gold Open Access which fosters wider collaboration and increased citations

- maximum visibility for your research: over 100M website views per year

At BMC, research is always in progress.

Learn more biomedcentral.com/submissions 